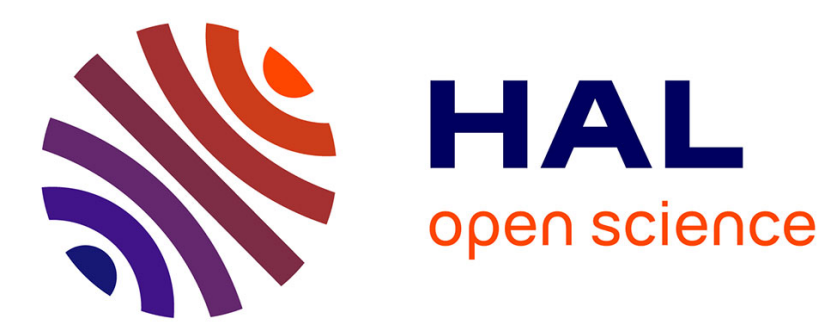

\title{
Design of a fluorinated magneto-responsive material with tuneable ultrasound scattering properties
}

Kévin Zimny, Benoit Mascaro, Thomas Brunet, Olivier Poncelet, Christophe Aristégui, Jacques Leng, Olivier Sandre, Olivier Mondain-Monval

\section{- To cite this version:}

Kévin Zimny, Benoit Mascaro, Thomas Brunet, Olivier Poncelet, Christophe Aristégui, et al.. Design of a fluorinated magneto-responsive material with tuneable ultrasound scattering properties. Journal of materials chemistry B, 2014, 2 (10), pp.1285-1297. 10.1039/c3tb21585g . hal-00948290

\section{HAL Id: hal-00948290 https://hal.science/hal-00948290}

Submitted on 24 Nov 2018

HAL is a multi-disciplinary open access archive for the deposit and dissemination of scientific research documents, whether they are published or not. The documents may come from teaching and research institutions in France or abroad, or from public or private research centers.
L'archive ouverte pluridisciplinaire HAL, est destinée au dépôt et à la diffusion de documents scientifiques de niveau recherche, publiés ou non, émanant des établissements d'enseignement et de recherche français ou étrangers, des laboratoires publics ou privés. 


\title{
Design of a fluorinated magneto-responsive material with tuneable ultrasound scattering properties
}

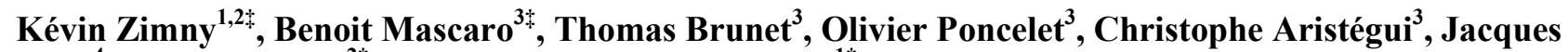 \\ Leng $^{4}$, Olivier Sandre ${ }^{2^{*}}$, and Olivier Mondain-Monval ${ }^{1^{*}}$
}

\author{
${ }_{5}$ Received 13th November 2013, Accepted 24th December 2013 \\ DOI: $10.1039 / \mathrm{C} 3 \mathrm{TB2} 2585 \mathrm{G}$
}

In this work, we describe the preparation of emulsions of fluorinated ferrofluid droplets suspended in a yield-stress hydrogel (Bingham fluid) with potential applications for ultrasound (US) spectroscopy and imaging. Fluorinated ferrofluids were obtained using an original multi-step process leading to the proper 10 suspension of magnetic nanoparticles (MNPs) coated by a layer of fluoroalkylsilane in fluorinated oil. The efficiency of the sol-gel coating reaction was followed by several methods including infrared and Xray photoelectron spectroscopy, small angle neutron scattering and magnetometry. The resulting suspension of silanized-MNPs behaves as a true fluorinated ferrofluid, remaining stable (i.e. a monophasic suspension of well dispersed MNPs) in magnetic inductions as high as $7 \mathrm{~T}$. These ferrofluids 15 were employed to prepare monodisperse emulsions in a Bingham gel using a robotic injection device. Using ultrasound spectroscopy, we show that the emulsion droplets behave as Mie-type acoustic waves resonators due to the high sound-speed contrast between the droplets and the matrix. When subjected to a magnetic field, the ferrofluid droplets elongate in the field direction, which in return modifies the acoustic response of the material. The resonance frequency peaks scale as the inverse of the emulsion droplet size 20 encountered by the wave propagation vector. These results might open a new road towards the realisation of ultrasound contrast agents guided by magnetic fields and with a tuneable attenuation spectrum.

\section{Introduction}

Possessing numerous original properties, fluorinated materials are used in a wide variety of applications such as coating agents for 25 cooking devices or fabrics, ion exchange membranes or as biomaterials for cardiovascular implants. ${ }^{1}$ They present low thermal conductivity, high mass density, chemical inertia, and unflammability. Thus, several companies developed low viscosity perfluoro- or semi-fluorinated oils with good biocompatibility for 30 applications such as blood substitutes, medical devices or ultrasound contrast agents, together with low environmental cost i.e. zero ozone-depletion and low greenhouse effect of their vapour in the atmosphere. ${ }^{2}$ The spreading in environment by industry of perfluoroalkanes motivated toxicological studies. If 35 perfluorooctanoic acid (PFOA) was shown to exhibit very slow clearance from living organisms, ${ }^{\mathbf{3 , 4}}$ as ascribed to the outstanding stability of carbon-fluorine bonds, other fluorinated chemicals are considered as "bio-safe". Among them, fluorinated ultrasound contrast agents (UCA) commonly referred to as "microbubbles" 40 are authorized for intravenous injection in humans under brand names as SonoVue ${ }^{\circledR}$, Optison ${ }^{\circledR}$, Imavis ${ }^{\circledR}$, Definity ${ }^{\circledR}$, Imagent ${ }^{\circledR} \ldots$

Due to their very low solubility in water (less than $10 \mathrm{ppm}$ ) and, on the contrary, to their high compressibility and ability to dissolve large quantities of gases $\left(\mathrm{O}_{2}, \mathrm{~N}_{2}, \mathrm{CO}_{2} \ldots\right),{ }^{5}$ volatile 45 fluorocarbons can indeed be used to fill the inner components of microbubbles. The UCA echogenicity relies on the impedance contrast between tissues mainly made of water $\left(Z_{\text {water }} \approx 1.5 \times 10^{6}\right.$ Rayl) and the materials to be injected, which is often a gas phase $\left(Z_{\text {air }} \approx 340\right.$ Rayl). However gas microbubbles also have a short 50 lifetime due to their physicochemical instability in a fluid and tendency to rapidly burst or to coalesce. One way to increase the lifetime of UCA in blood is to mix air with a perfluorocarbon gas that presents very low solubility in water, thus acting as "osmotic agent" and slowing down the Ostwald ripening process. 5 Several 55 routes were developed to obtain longer-lasting UCA, on the one hand by coating the bubbles with a stabilising shell of lipids or polymers, ${ }^{6,7}$ on the other hand by adding to air or nitrogen a partial pressure of fluorinated gas, in that case wrapped by a shell of hydrogenated ${ }^{8}$ or F-alkylated double-tailed phospholipids.' ${ }_{60}$ Typical volatile fluoroalkanes incorporated in microbubbles are octafluoropropane, ${ }^{\mathbf{1 0}}$ decafluorobutane, ${ }^{11}$ tetradecafluorohexane (commercialised by $3 \mathrm{M}$ as the Fluorinert ${ }^{\mathrm{TM}}$ FC-72 reference), ${ }^{\mathbf{8},} \mathbf{9}$ or perfluorooctylbromide (PFOB). ${ }^{7}$ Recently, several teams reported the decoration of the surface of microbubbles by iron 65 oxide nanoparticles, both for pure air $^{12}$ and for mixed air/fluorocarbon gas bubbles. ${ }^{\mathbf{1 0}} \mathbf{1 3}$ The idea was to be able to guide such magnetic microbubbles against the strong flow-rate of blood circulation by the use of a magnetic field gradient.

Thus, the US imaging community is still in search for 70 alternatives to gas bubbles, which present a high echogenicity but poor long term stability. We propose here a new type of materials 
made of fluorinated ferrofluid oil droplets exhibiting both a large sound-speed contrast $(\sim 1 / 3)$ with aqueous media $\left(500 \mathrm{~m} \cdot \mathrm{s}^{-1}\right.$ / $1500 \mathrm{~m} \cdot \mathrm{s}^{-1}$ ) and sensitivity to an external magnetic fluid. When dispersed in an aqueous yield-stress matrix, magnetic fluorinated 5 oil droplets are not only magnetically guidable but also exhibit strong Mie resonances at specific frequencies, ${ }^{\mathbf{1 4}}$ varying with the intensity of an applied magnetic field and its orientation with respect to the wave propagation vector. Such magneto-responsive attenuation properties are described precisely in a companion 10 article. $^{15}$ The present manuscript deals with chemistry and softmatter methods necessary to build this tuneable acoustic material. First, we describe the process used to synthesise the magnetic nanoparticles and to coat them with a layer of perfluorosilane. This fluorinated ferrofluid is then fully characterised by different 15 spectroscopic, microscopic and magnetic methods. At last, we present the fabrication of a monodisperse emulsion in an aqueous yield-stress fluid and an example of tuneable acoustic properties.

Since decades, sol-gel methods have opened a golden gate towards the so-called "soft chemistry" which enables to obtain 20 organic-inorganic nano-sized or nano-structured hybrid materials based on silica exhibiting a wide variety of structures and morphologies, and more recently on metal-oxide frameworks. ${ }^{16}$ Organosilanes are commonly used to functionalise silica surfaces by a hydrolysis-condensation mechanism initiated at the 25 inorganic surface, that is rich in silanol moieties ( $\mathrm{Si}-\mathrm{OH})$ arising from the hydrated state of the solid surface. Monolayers of semifluorinated alkylsilanes are chosen for instance to render glass surfaces highly hydrophobic. ${ }^{17,18}$ The same reactions exist for the hydrated surface of iron oxide (Fe-OH groups), but they have 30 been much less developed. The chemical grafting of molecules onto iron oxides often starts by the coating with a molecular monolayer of (3-aminopropyl)triethoxysilane (APTS). ${ }^{19-27}$ This short molecule is too small to bring enough electrosteric repulsions between particles to confer any colloidal stability to
35 the MNPs in water, but they provide amino groups enabling further functionalisation steps. Several studies involve a ligandexchange process starting from an initial oleic acid monolayer that is replaced by the organosilane. ${ }^{\mathbf{2 6}, \mathbf{2 8 - 3 0}}$ Apart from APTS, other organosilanes were grafted onto the surface of iron oxide ${ }_{40}$ MNPs, such as (3-glycidyloxypropyl)trimethoxysilane, ${ }^{31}$ cyanoethyltrimethoxysilane, $^{32}$ or 2-bromo-2-methyl-N-(3(triethoxysilyl)propyl) propanamide to initiate polymer chain growth by a "grafting-from" controlled polymerisation method. ${ }^{33}$ In almost all the previous works, silanization was an intermediate 45 step needed to anchor a molecular shell around the magnetic cores by covalent bonds, which is preferable than most common adsorption routes with chelating ligands such as carboxylic acids, phosphates, sulfonates or catechols. Only macromolecular silanes such as triethoxy(methoxypolyethyleneoxy)silane ${ }^{\mathbf{2 9}}$ or 50 trifluoroethylester-poly(ethyleneglycol) ${ }^{\mathbf{3 0}}$ were shown to achieve direct colloidal stabilisation of MNPs in water before any further coupling reaction.

\section{Approach developed here}

In this study, we propose to accomplish the chemical grafting of ${ }_{55} 1 \mathrm{H}, 1 \mathrm{H}, 2 \mathrm{H}, 2 \mathrm{H}-$ perfluorodecyltriethoxysilane (PFDTS) leading to stable colloidal suspensions of iron oxide MNPs in fluorinated oils. The goal for this magnetic fluid is to remain monophasic whatever the strength of an applied magnetic field, which is the definition of a fluorinated "true ferrofluid" (as opposed to a 60 magneto-rheological fluid, where the MNPs make dipolar chains under a magnetic field). Due to the insolubility of perfluorinated molecules in hydrogenated solvents, we propose a route (Scheme 1) starting by adsorption of a perfluoropoly(ether) carboxylic acid (PFPE-COOH, Krytox ${ }^{\mathrm{TM}}$ 157-FSH, Dupont) onto the positively 65 charged surface of iron oxide followed by ligand-exchange and silanization with PFDTS, leading to stable fluorinated ferrofluids.

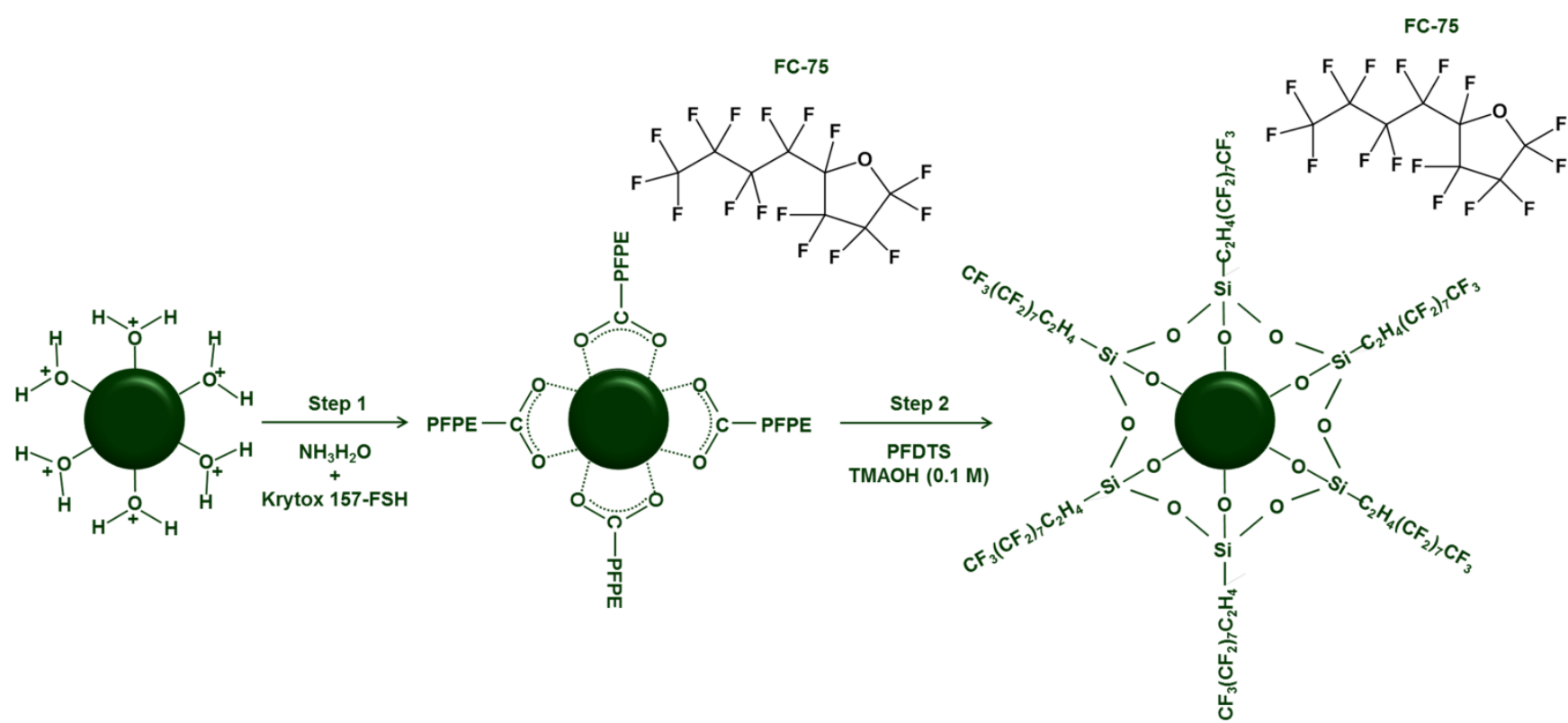

Scheme 1 Fluorinated ferrofluid prepared in two steps. The proper dispersion of iron oxide nanoparticles in a fluorinated oil involves: Step 1: adsorption of a perfluoropoly(ether) surfactant (Krytox ${ }^{\mathrm{TM}}$ 157-FSH) onto the nanoparticles and phase transfer into a fluorinated oil such as perfluoro-270 butyltetrahydrofuran (Fluorinert ${ }^{\mathrm{TM}}$ FC-75); Step 2: ligand exchange, and then chemical grafting (sol-gel reaction) of 1H,1H,2H,2Hperfluorodecyltriethoxysilane (PFDTS) directly onto the hydroxyl groups of the iron oxide surface. The two steps were performed in alkaline media (respectively ammonium hydroxide and tetramethylammonium hydroxide). 


\section{Experimental section}

\section{Preparation of a fluorinated ferrofluid}

\section{Synthesis of magnetic iron oxide nanoparticles}

Magnetic nanoparticles (MNPs) were prepared by coprecipitation 5 of iron $^{+ \text {II }}$ and iron ${ }^{+I I I}$ salts in alkaline media followed by surface charge reversal in nitric acid and oxidation with iron nitrate according to Massart procedure. ${ }^{34}$ At first, $180 \mathrm{~g}$ of $\mathrm{FeCl}_{2} \cdot 4 \mathrm{H}_{2} \mathrm{O}$ were mixed with $100 \mathrm{~mL}$ of $\mathrm{HCl}(37 \%)$ and $500 \mathrm{~mL}$ of deionised water. The mixture was stirred until complete dissolution of ${ }_{10} \mathrm{FeCl}_{2}$. After addition of $365 \mathrm{~mL}$ of $\mathrm{FeCl}_{3}(45 \%)$ and $2 \mathrm{~L}$ of deionized water, $1 \mathrm{~L}$ of ammonia solution (28\%) was added rapidly under vigorous stirring $(800 \mathrm{rpm})$. The obtained magnetite particles were then collected after sedimentation on a strong magnet. After precipitate washing with water, $360 \mathrm{~mL}$ of 15 nitric acid and $2 \mathrm{~L}$ of water were added. Then, $323 \mathrm{~g}$ of $\mathrm{Fe}\left(\mathrm{NO}_{3}\right)_{3} \cdot 9 \mathrm{H}_{2} \mathrm{O}$ was added with $800 \mathrm{~mL}$ of water in order to oxidise the magnetite particles $\left(\mathrm{Fe}_{3} \mathrm{O}_{4}\right)$ into maghemite $\left(\gamma-\mathrm{Fe}_{2} \mathrm{O}_{3}\right)$. After sedimentation and washing, the addition of water leads to the formation of a stable aqueous ferrofluid. The obtained 20 magnetic nanoparticles are dispersed in water due to their positive surface charge in acidic conditions. The synthesised nanoparticles were separated into two fractions of hydrodynamic sizes, respectively $D_{\mathrm{H}}=11 \mathrm{~nm}(P D I=0.013)$ and $D_{\mathrm{H}}=36 \mathrm{~nm}$ $(P D I=0.024)$ following a size-sorting procedure based on the 25 phase-separation by addition of electrolyte in excess to screen electrostatic repulsions. ${ }^{35}$ The smaller size fraction (SP aqueous ferrofluid) was used mainly for the study of the fluorinated coating, while the larger size fraction (LP aqueous ferrofluid) enabled preparing magnetic emulsions in water-based gels with 30 larger magnetic susceptibility and thus higher deformations of the drops for a given magnetic field.

\section{Fluorinated surfactant coating}

At first, the MNPs were coated with a high molecular weight (7500 $\mathrm{g} \cdot \mathrm{mol}^{-1}$ nominal, $\sim 5000-6000 \mathrm{~g} \cdot \mathrm{mol}^{-1}$ measured $^{36}$ ) 35 perfluoropoly(ether) end-functionalised with a carboxylic acid group (PFPE-COOH, Krytox ${ }^{\text {TM }}$ 157-FSH, Dupont), which enables transferring them from water to perfluoro-2-butyltetrahydrofuran (Fluorinert $^{\mathrm{TM}}$ FC-75, 3M) (see step 1 on Scheme 1). Krytox ${ }^{\mathrm{TM}}$ 157-FSH was first mixed with ammonium hydroxide to form a 40 carboxylate salt. The aqueous ferrofluid was afterwards directly added to this mixture (molar ratio $\left.n\left(\gamma-\mathrm{Fe}_{2} \mathrm{O}_{3}\right) / n(\mathrm{PFPE})=30\right)$. In such conditions, the deprotonated carboxylic head-groups of Krytox $^{\mathrm{TM}}$ molecules bear a negative charge and adsorb onto the positively charged iron oxide surface. The obtained system was 45 then washed several times with acetone in order to separate the Krytox ${ }^{\mathrm{TM}}$-covered MNPs from the polar mixture. Then, ethanol was added and the precipitate was collected after sedimentation on a strong permanent magnet. After three steps of washing with ethanol and acetone, fluorinated oil FC-75 was added to achieve a 50 suspension around $4 \mathrm{wt} \%$ and the residual solvents were eliminated by heating 1 hour at $353 \mathrm{~K}$ in an oven. After this drying step, the ferrofluid is referred to as "surfacted ferrofluid". These surfacted-ferrofluids present a high viscosity $(>0.1 \mathrm{~Pa} \cdot \mathrm{s})$ incompatible with deposition through capillaries. This high 55 viscosity is due to the relatively high viscosity of the FC75/Krytox ${ }^{\mathrm{TM}}$ continuous phase. In addition, the interfacial tension $\gamma$ between the ferrofluid and a dilute Carbopol $^{\mathrm{TM}}$ solution ( $\gamma=25 \mathrm{mN} \cdot \mathrm{m}^{-1}$ as measured using a pendant drop experiment on a pendant and sessile drop tensiometer Krüss ${ }^{\mathrm{TM}}$ DSA100) seems 60 too high to observe a deformation of the droplets under a magnetic field of reasonable intensity (i.e. of induction $B$ less than $60 \mathrm{mT}$ ).

\section{Ligand exchange and silanization}

A second step of ligand exchange was performed in order to 65 replace Krytox ${ }^{\text {TM }}$ 157-FSH surfactants by organofluorosilanes, with the purpose to graft them covalently onto the MNPs (step 2 on Scheme 1). The chosen fluoroalkylsilane, $1 \mathrm{H}, 1 \mathrm{H}, 2 \mathrm{H}, 2 \mathrm{H}-$ perfluorodecyltriethoxy-silane (PFDTS, Sigma-Aldrich) was added in large excess $(n(\mathrm{PFDTS}) / n(\mathrm{PFPE})=12)$ directly to the 70 surfactant-based ferrofluid in FC-75 (or another oil from the Fluorinert ${ }^{\mathrm{TM}}$ series such as the less volatile FC-40). It was first hydrolysed and then condensed around the MNPs via a sol-gel process. The reaction occurred under basic conditions with tetramethylammonium hydroxide (TMAOH, Sigma, $0.1 \mathrm{M}$ ) as 75 catalyst. The mixture was placed under stirring $(300 \mathrm{rpm})$ for 3 days at $343 \mathrm{~K}$. The sample was washed several times with water, ethanol and acetone then isolated on a magnet in order to remove the un-reacted silane. MNPs were finally dried in a vacuum oven during 1 hour at $353 \mathrm{~K}$ and afterward dispersed in fluorinated oil 80 FC-75 at a concentration of $300 \mathrm{~g} \cdot \mathrm{L}^{-1}$, representing volume and weight fractions of respectively $6 \mathrm{vol} \%$ (15 wt $\%)$ of iron oxide according to the mass densities (respectively $5 \mathrm{~g} \cdot \mathrm{cm}^{-3}$ for $\gamma-\mathrm{Fe}_{2} \mathrm{O}_{3}$ and $1.77 \mathrm{~g} \cdot \mathrm{cm}^{-3}$ for the fluorinated oil FC-75). After this second step, the ferrofluid referred to as "silanized ferrofluid" presents ${ }_{85}$ low values of viscosity and interfacial tension with a dilute solution of Carbopol ${ }^{\mathrm{TM}}$ in water $\left(\gamma=11 \mathrm{mN} \cdot \mathrm{m}^{-1}\right.$ as measured by a pendant drop experiment).

\section{Preparation of the monodisperse magnetic emulsion}

\section{Aqueous gel}

90 A yield-stress (Bingham) fluid was prepared by mixing $0.5 \mathrm{~g}$ of ramified sodium poly(acrylate) $\left(\right.$ Carbopol $\left.^{\mathrm{TM}} 2050 \mathrm{ETG}\right)$ with 100 $\mathrm{mL}$ of deionised water at $323 \mathrm{~K}$. After 30 minutes of stirring at $170 \mathrm{rpm}$, the mixture was aged at room temperature during 40 min. Finally, $0.5 \mathrm{~mL}$ of $\mathrm{NaOH}$ at $10 \mathrm{M}$ was added under vigorous 95 stirring until $\mathrm{pH}$ reached $7 \pm 0.5$. The gel was then diluted with deionised water to obtain a range of concentration comprised between $0.05 \%$ and $0.5 \% \mathrm{w} / \mathrm{w}$ then centrifuged at $6000 \mathrm{rpm}$ in order to remove bubbles. Rheological measurements were performed on an AR2000 TA Instruments rheometer to determine 100 the yield stress at different concentrations. The magnetic emulsions described in this paper were prepared in Carbopol ${ }^{\mathrm{TM}}$ gels at $0.05 \% \mathrm{w} / \mathrm{w}$. In this case, the rheological curve exhibits a yield stress of $1.5 \mathrm{~Pa}$. 


\section{Injection of ferrofluid in gel to prepare the emulsion}

Using robotics, the fluorinated ferrofluids were injected through silica capillaries (coated by polyimide) directly into the aqueous gel. Silica capillaries with respective internal diameter (ID)/outer s diameter (OD) of 40/105 $\mu \mathrm{m}$ and $75 / 150 \mu \mathrm{m}$ purchased from Polymicro $^{\mathrm{TM}}$ Technologies were inserted in corresponding sleeves of $\mathrm{OD}=1 / 16$ " (respectively F-237 and F-238, Upchurch ${ }^{\mathrm{TM}}$ ). The sleeve was inserted in a 10-32 PEEK nut with a 1/16" OD ferrule $\left(\right.$ Upchurch $\left.^{\mathrm{TM}}\right)$ connected through a P-659 Luer-lock to a syringe. 10 As previously done for bubbly media ${ }^{37}$ or fluorinated oil droplets, ${ }^{14,} 38$ periodic rows of regularly spaced fluorinated ferrofluid droplets were deposited by a motorised motion of the capillary relatively to the gel. The magnetic fluorinated oil was continuously pushed into the aqueous gel by a $0-1.8$ bar pressure 15 generator (Elveflow ${ }^{\mathrm{TM}}$ AF1, Elvesys ${ }^{\circledR}$, Paris, France). Motorised displacements of the plate led to the formation of aligned droplets. Samples made of series of drop lines suspended in the hydrogel were then transferred into the measurement cell $(4 \times 4 \times 3$ $\mathrm{cm}^{3}$ ) placed inside an electromagnet made of two solenoids (1000 20 turns each) connected in serial with a U-shape soft iron polar piece and an air gap of $4 \mathrm{~cm}$. The magnetic induction $B$ was measured by a LakeShore ${ }^{\mathrm{TM}} 425$ Hall-probe gaussmeter.

\section{Analytical methods}

Transmission electron microscopy (TEM)

25 Transmission electron microscopy micrographs were recorded on a Hitachi H7650 microscope working at $80 \mathrm{kV}$. Samples were diluted at $\sim 0.2 \mathrm{~g} \cdot \mathrm{L}^{-1}$ in the volatile perfluorohexane (Fluorinert ${ }^{\mathrm{TM}}$ FC-72) and sprayed onto the grids. The images were analysed by automated particle counting after threshold and watershed filters 30 using the ImageJ software (http://rsbweb.nih.gov/ij/).

\section{Infrared spectroscopy (ATR IR)}

Infrared measurements were performed on a Bruker Tensor 27 FT-IR spectrometer using the Diamond attenuated total reflection method. Samples were analysed after drying during $1 \mathrm{~h}$ in a 35 vacuum oven at $353 \mathrm{~K}$. The IR spectra were recorded in the wave number range $800-4000 \mathrm{~cm}^{-1}$.

\section{X-ray photoelectron spectroscopy (XPS)}

A VG Scientific 220 i-XL ESCALAB spectrometer was used for the MNPs surface analysis (at a maximal depth of $10 \mathrm{~nm}$ ) with a 40 non-monochromatised $\mathrm{MgK}_{\alpha}$ source $(h v=1253.6 \mathrm{eV})$ at $130 \mathrm{~W}$ $(13 \mathrm{kV}$ and $10 \mathrm{~mA})$. A pressure of $10^{-7} \mathrm{~Pa}$ was maintained in the chamber during analysis. The analysed area was about $150 \mu \mathrm{m}$ in diameter. The full spectra $(0-1150 \mathrm{eV})$ were obtained with constant pass energy of $150 \mathrm{eV}$ and high resolution spectra at 45 constant pass energy of $40 \mathrm{eV}$. Charge neutralisation was required for all insulating samples. The peaks were referenced to $\mathrm{Si}_{2 \mathrm{p}}$ maximum shifted at $103.5( \pm 0.1) \mathrm{eV}$. High resolution spectra were fitted and quantified using the AVANTAGE software provided by ThermoFisher Scientific.

\section{${ }_{50}$ Dynamic Light Scattering (DLS)}

DLS measurements were performed using a nanoparticle size analyser Vasco $^{\mathrm{TM}}$ DL135 (Cordouan Technologies, Pessac, France) equipped with a diode laser operating at a $650 \mathrm{~nm}$ wavelength at $30 \%$ of full power, and a photodiode detector 55 collecting backscattered light at an angle of $135^{\circ}$. For each sample, intensity measurements were carried out at $298 \mathrm{~K}$ in a multi-acquisition mode implying 20 correlograms. Nanoparticles $Z$-average diameters and polydispersity indexes $(P D I)$ were obtained by fitting each correlogram with the $2^{\text {nd }}$ order ${ }_{60}$ Cumulants algorithm. The time interval and number of channels were fixed for each measurement at 11 and $1000 \mu$ s respectively. Size distribution histograms were generated after each acquisition using the Pade-Laplace inversion algorithm. After accumulation of 20 curves, the obtained histograms were fitted with a log65 normal function. Hydrodynamic size histograms obtained by the Cumulants and Pade-Laplce analyses were compared.

\section{Volume fraction measurements}

At first, MNPs were diluted, either in dilute $\mathrm{HNO}_{3}$ (at $\mathrm{pH}$ 2) for aqueous ferrofluids or fluorinated oils for the fluorinated 70 ferrofluids (FC-40 or FC-75). They were then analysed by UVVis. UV-Vis spectra were recorded in quartz cuvettes on a Molecular devices spectrophotometer SpectraMax ${ }^{\mathrm{TM}}$ between 240 and $800 \mathrm{~nm}$ by step of $4 \mathrm{~nm}$. Molar concentrations were determined by fitting a calibration curve $\mathrm{e}^{\mathbf{3 9}, 40}$ and converted into 75 volume fractions using the tabulated mass densities. Another method based on direct mass density measurement was employed for highly concentrated suspensions for which the volume was impossible to control with a micropipette. In that case, a plastic tubing of given length and diameter was simply weighted using a 80 precision balance in order to determine the mass density. Then, the MNPs volume fraction $\phi$ was computed from the apparent mass density $\rho=\phi \cdot \rho_{\text {iron oxide }}+(1-\phi) \cdot \rho_{\text {FC-75 }}$ where $\rho_{\text {iron oxide }}=5$ $\mathrm{g} \cdot \mathrm{cm}^{-3}$ and $\rho_{\mathrm{FC}-75}=1.77 \mathrm{~g} \cdot \mathrm{cm}^{-3}$ are the mass densities of iron oxide and of the FC-75 oil, respectively.

${ }_{85}$ Small angle neutron scattering (SANS)

SANS curves were recorded on the PACE spectrometer at the LLB-CEA Saclay neutron facility, France. The scattering wave vector $q$ ranged from $0.00243 \AA^{-1}$ to $0.37 \AA^{-1}$ thanks to the use of 3 different configurations: "small- $q$ range" (sample-to detector 90 distance $D=4.7 \mathrm{~m}$, neutron wavelength $\lambda=17 \AA$ ), "medium- $q$ range" $(D=3 \mathrm{~m}$ and $\lambda=6 \AA)$, and "large-q range" $(D=1 \mathrm{~m}$ and $\lambda=6 \AA)$. The samples analysed in $1 \mathrm{~mm}$-thick quartz cuvettes exhibited low incoherent background do to their low hydrogen content. The curves were converted into absolute intensity unit $95\left(\mathrm{~cm}^{-1}\right)$ using calibration by standards (subtraction by empty cell and normalisation by signal of $\mathrm{H}_{2} \mathrm{O}$ to take into account the efficiency of the detector). ${ }^{41}$ The SANS curves were fitted with a polydisperse core-shell form factor using the SASView program available at http://www.sasview.org/. The values of neutron 100 scattering length density (SLD) were calculated using chemical formulas and tabulated values of the atomic neutron scattering lengths. These SLD values were $6.98,2.1$, and $3.84 \times 10^{10} \mathrm{~cm}^{-2}$ respectively for $\gamma-\mathrm{Fe}_{2} \mathrm{O}_{3}$, PFDTS, and FC-75.

Superconducting quantum interference device (SQUID)

105 Magnetisation curves were recorded at room temperature using a Quantum Design $^{\text {TM }}$ MPMSXL SQUID magnetometer from 0 to 7 T. MNPs were diluted at $1 \% \mathrm{v} / \mathrm{v}$ in $\mathrm{HNO}_{3} \mathrm{pH} 2$ for aqueous ferrofluids or in FC-75 for fluorinated ferrofluids. A wet mass around $1 \mathrm{mg}$ of each sample (including solvent) was weighted 110 precisely in an impermeable bag sealed at the end of a plastic straw inserted inside the magnet hole. The diamagnetic contribution of the solvent was fitted by a negative slope line (dominant at high field) subtracted from the total curve to yield the contribution of the MNPs only.

\section{${ }_{115} 3$ Results and discussion}




\section{Sizes and colloidal stability of MNPs in fluorinated oils}

The size and colloidal stability in fluorinated oils was assessed by DLS (Table 1). Two kinds of fluorinated nanoparticles were prepared in this work. They were noted 'surfacted' after coating 5 with the Krytox ${ }^{\mathrm{TM}}$ surfactant (step 1 on Scheme 1) and 'silanized' after grafting with the PFDTS silane (step 2 on Scheme 1). Sample 1 was prepared from an aqueous ferrofluid of larger nanoparticles noted 'aqueous LP' and used to produce magnetic emulsions in gels whereas Sample 2 was prepared from 'aqueous ${ }_{10} \mathrm{SP}$ ' and used for the SANS experiment described further. The different size measurements performed on the two samples at the various steps of the coating procedure are given in Table 1.

Table 1 Hydrodynamic (DLS) and physical (TEM) diameters of the nanoparticles for several prepared ferrofluids

\begin{tabular}{|c|c|c|c|c|c|}
\hline \multicolumn{6}{|c|}{ LP (Sample 1) } \\
\hline & \multicolumn{2}{|c|}{ Cumulants $^{a}$} & \multicolumn{2}{|c|}{ Pade-Laplace $^{b}$} & TEM analysis \\
\hline & $\begin{array}{l}Z \text {-ave } \\
\text { (nm) }\end{array}$ & PDI & $\begin{array}{l}<D> \\
(\mathrm{nm})\end{array}$ & $C V(\%)$ & $\begin{array}{c}<D> \pm \Delta D \\
\quad(\mathrm{~nm})\end{array}$ \\
\hline Aqueous & 36.5 & 0.024 & 35.9 & 6.9 & $\mathrm{NM}^{c}$ \\
\hline Surfacted & 68 & 0.07 & 79.3 & 135 & $\mathrm{NM}^{c}$ \\
\hline Silanized & 51 & 0.05 & 51.5 & 6.4 & $9.3 \pm 3.4$ \\
\hline \multicolumn{6}{|c|}{ SP (Sample 2) } \\
\hline & \multicolumn{2}{|c|}{ Cumulants $^{a}$} & \multicolumn{2}{|c|}{ Pade-Laplace $^{b}$} & TEM \\
\hline & $\begin{array}{c}Z \text {-ave } \\
\text { (nm) }\end{array}$ & PDI & $\begin{array}{l}<D> \\
(\mathrm{nm})\end{array}$ & $C V(\%)$ & $\begin{array}{c}<D> \pm \Delta D \\
\quad(\mathrm{~nm})\end{array}$ \\
\hline Aqueous & 11 & 0.013 & 10.1 & 11.2 & $\mathrm{NM}^{c}$ \\
\hline Surfacted & 91.5 & 0.09 & 99.1 & 79.6 & $5.8 \pm 2.6$ \\
\hline Silanized & 56.2 & 0.065 & 57.3 & 8.6 & $7.3 \pm 2.3$ \\
\hline
\end{tabular}

${ }_{15}{ }^{a} 2^{\text {nd }}$ order cumulant fit ( $Z$-average diameter and polydispersity index) and ${ }^{b}$ Pade-Laplace fit (mean diameter, coefficient of variation) of the DLS correlograms for aqueous, Krytox ${ }^{\mathrm{TM}}$-'surfacted' and PFDTS- 'silanized' ferrofluids. Sample 1 was prepared from the large fraction (aqueous LP). Sample 2 was prepared from the smaller size fraction (aqueous SP). $20^{c} \mathrm{NM}$ : not measured.

The two methods used to analyse the DLS data lead to roughly the same average particles diameters and broadness of the distribution (the values of $P D I$ and $C V$ being clearly correlated). After step 1 (coating with Krytox ${ }^{\mathrm{TM}}$ ), the 'surfacted' nanoparticles 25 remain dispersed and stable but exhibit a significant size and sizedispersity increase ( $Z$-average hydrodynamic diameters respectively of 68 and $92 \mathrm{~nm}$ for the two batches LP and SP, PDI respectively 0.07 and 0.09 ). This result indicates that the MNPs tend to aggregate when coated with the fluorinated surfactant 30 $\mathrm{Krytox}^{\mathrm{TM}}$ 157-FSH. After step 2 (chemical grafting of perfluorinated organosilane at the particles surface), one observes a decrease of the sizes of MNPs and of the polydispersity index that reveals an improved colloidal stability and a narrower size distribution. The mean diameter of the MNPs and its coefficient 35 of variation $(\mathrm{CV})$, both calculated by fitting the Pade-Laplace histogram with a log-normal distribution law, show the same evolution. The results exhibit a large $C V$ difference between surfacted and grafted ferrofluids. Figure 1 shows the evolution of the hydrodynamic diameter for three different samples before and 40 after grafting with PFDTS. In all cases, each 'silanized' ferrofluid presents a lower hydrodynamic diameter and a sharper distribution than the corresponding 'surfacted' ferrofluid.

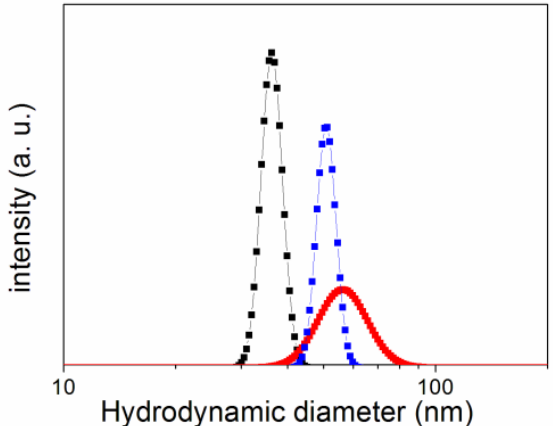

a)

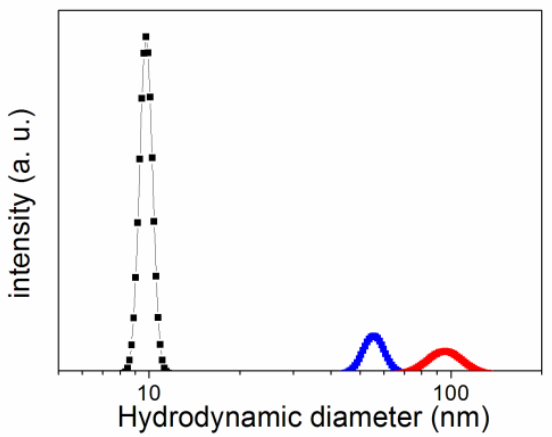

b)

45 Fig. 1 Evolution of the hydrodynamic diameter at different steps of the synthesis obtained by fitting the Pade-Laplace histograms with lognormal laws. Aqueous (black line), 'surfacted' (red line) and 'silanized' ferrofluids (blue line) for a) Sample 1 (LP), and b) Sample 2 (SP).

\section{Transmission electron microscopy (TEM)}

${ }_{50}$ The MNPs were also directly imaged by TEM (Fig. 2). The sizes of the MNPs deduced by image analysis of these pictures are given in Table 1.

The sizes by TEM are much smaller than those deduced by DLS, which indicates that the magnetic cores observed 55 individually on the TEM pictures form clusters of several nanoparticles when suspended in a liquid. To represent the sizedispersity, the histograms of the diameters $d$ obtained by DLS (Fig. 1) or TEM (Fig. 2) were all fitted by a log-normal distribution law of median value $d_{0}$ and characteristic broadness ${ }_{60} \sigma$, as written below.

$$
P(d)=\frac{1}{\sqrt{2 \pi} \sigma d} \exp \left[-\frac{1}{2 \sigma^{2}}\left(\ln \frac{d}{d_{0}}\right)^{2}\right]
$$

On a logarithmic scale such as the horizontal axis of Fig. 1, this distribution looks like a Normal law, $\sigma$ being the standard deviation of $\ln (d)$. Then, the moments of the distribution can be ${ }_{65}$ easily calculated. In particular the number-averaged $\left(d_{\mathrm{n}}\right)$ and volume-averaged $\left(d_{\mathrm{w}}\right)$ diameters are obtained respectively by following formulas.

$$
\begin{gathered}
d_{n}=<d>=d_{0} \cdot \exp \left(\sigma^{2} / 2\right) \\
d_{w}=<d^{4}>/<d^{3}>=d_{0} \cdot \exp \left(7 \sigma^{2} / 2\right)
\end{gathered}
$$


a)



b)

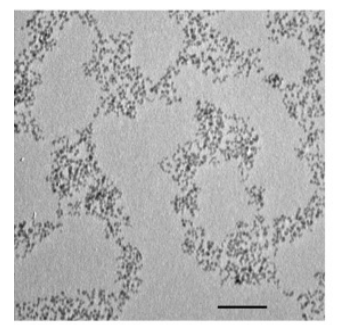

c)
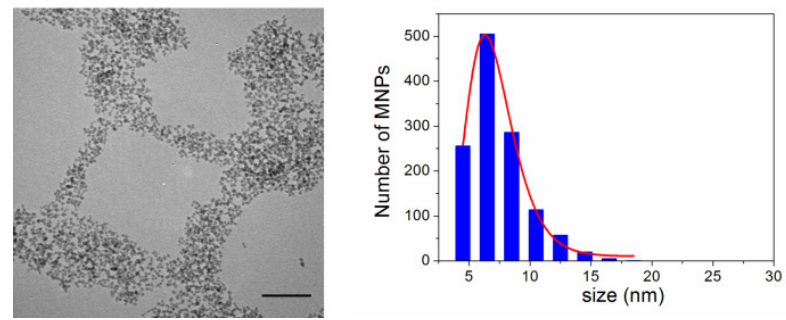

Fig. 2 TEM micrographs and corresponding size histograms deduced from image analysis and fitted with a log-normal function. a) 'Silanized' sample 1 (LP, $9.3 \pm 3.4 \mathrm{~nm}$ ); b) 'Surfacted' sample 2 (SP, $5.8 \pm 2.6 \mathrm{~nm}$ ); 5 c) 'Silanized' sample $2(\mathrm{SP}, 7.3 \pm 2.3 \mathrm{~nm})$. Scale bars represent $100 \mathrm{~nm}$.

For example, 'silanized' sample $1(\mathrm{LP})$ has $d_{\mathrm{n}}^{\text {TEM }}=10.2 \mathrm{~nm}$ and $d_{\mathrm{w}}{ }^{\text {TEM }}=19.1 \mathrm{~nm}$. At larger magnification (Fig. 3), the TEM micrographs evidence the presence of a shell around the nanoparticles, which has a lower electron density than iron oxide.

10 The size of this shell can be estimated at approximately $3 \mathrm{~nm}$ for sample 1 (Fig. 3). These observations are consistent with the formation of multi-layers of fluorinated silica around the MNPs. With sample 2 (from the aqueous SP batch), the size of the MNPs goes from around $5.8 \mathrm{~nm}$ before grafting to $7.3 \mathrm{~nm}$ after grafting.

15 The $1.5 \mathrm{~nm}$ difference can be assigned to the formation of a silica shell around the MNPs.

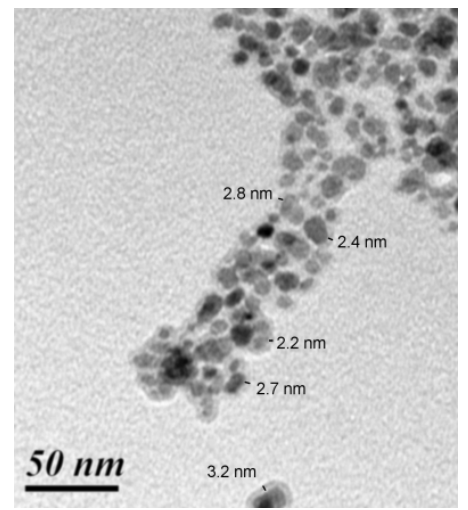

Fig. 3 High resolution TEM micrographs of the 'silanized' sample 1 (LP). The scale bar represents $50 \mathrm{~nm}$. The thickness of the silica-rich shell is 20 measured at several locations on this image.

\section{Small angle neutron scattering (SANS)}

The effect of the different coatings on the colloidal stability of the MNP dispersion in FC-75 oil was further studied by SANS with the nanoparticles of smaller sizes (sample 2). The intensity curve 25 of the PFDTS-silanized MNPs (Fig. 4) was well fitted by the form factor of a polydisperse suspension of core-shell particles modelled by an inorganic core radius $R_{0}=2.34 \mathrm{~nm}$ (Log-normal dispersion of standard width $\sigma=0.33$, SLD of $\gamma-\mathrm{Fe}_{2} \mathrm{O}_{3}$ ) wrapped by a silicon-rich shell of thickness $t_{0}=1.45 \mathrm{~nm}$ (polydispersity ${ }_{30} \sigma=0.64$, SLD of PFDTS) suspended in a medium with the theoretical SLD of the FC-75 oil. Near the lowest scattering vectors $q$, the Guinier approximation enables to measure a radius of gyration $R_{\mathrm{G}}=5.1 \mathrm{~nm}$ compatible with individually dispersed MNPs. On the contrary, the curve for the Krytox ${ }^{\mathrm{TM}}$-surfacted 35 ferrofluid could not be fitted simply by a core-shell form factor, presumably due to the contribution of cross-particle correlations (structure factor due to attractions). In that case, the Guinier plot leads to a gyration radius $R_{\mathrm{G}}=29 \mathrm{~nm}$, attesting the presence of finite sized aggregates of MNPs. From these SANS experiments, 40 we deduce that the grafting of a fluoroalkylsilane onto the MNPs greatly improved the quality of the dispersion in the fluorinated oil compared to mere adsorption of a fluorinated surfactant.

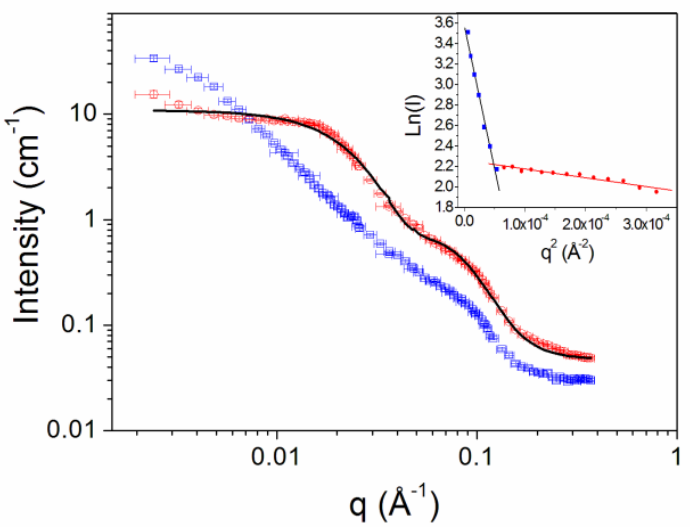

Fig. 4 SANS curves of the Krytox ${ }^{\mathrm{TM}}$-surfacted ferrofluid (blue markers), ${ }_{45}$ PFTDS-silanized MNPs (red markers) and fit by a polydisperse core-shell form factor (solid line). The volume fractions $\phi$ are respectively $0.5 \%$ and $3.4 \%$. Inset: Guinier plots of $\ln (I(q))$ as a function of $q^{2}$ enabling to measure the gyration radii $R_{\mathrm{G}}$ from the slope $-R_{\mathrm{G}}{ }^{2} / 3$.

\section{Spectroscopic study of the chemical grafting onto iron oxide}

\section{${ }_{50}$ ATR IR spectroscopy}

The efficiency of the grafting reaction was assessed by FT-IR spectroscopy. We display the spectra in three different regions (800-1400 $\mathrm{cm}^{-1}, 1500-2000 \mathrm{~cm}^{-1}$, and 2500-4000 $\mathrm{cm}^{-1}$ ) in order to ease the discussion on all the information that can be deduced.

55 Evidence for the surfactant adsorption at the surface of the MNPs (step 1) can be deduced from the comparison of the spectra of pure Krytox ${ }^{\mathrm{TM}} 157-\mathrm{FSH}$ and of the Krytox ${ }^{\mathrm{TM}}$-surfacted ferrofluid (Fig. 5: Spectra A and B). The IR spectrum of pure Krytox $^{\text {TM }} 157-$ FSH contains a large band at $980 \mathrm{~cm}^{-1}$ (band (a) on

${ }_{60}$ Fig. 5) characteristic of the stretching of $\mathrm{CF}_{3}$ bonds in the fluoropropylene oxide groups of the perfluoro-poly(ether) backbone. $^{42}$ The broad band at $1117 \mathrm{~cm}^{-1}$ is ascribed to $\mathrm{C}-\mathrm{O}$ stretching mode (b). Absorption bands at 1180, 1200 and 1230 $\mathrm{cm}^{-1}$ correspond to different stretching modes of $\mathrm{CF}_{\mathrm{x}}$ groups (c). 
The band at $1780 \mathrm{~cm}^{-1}$ (d) is assigned to carbonyl stretching of carboxylic acid. ${ }^{43}$ For Krytox ${ }^{\mathrm{TM}}$-surfacted ferrofluid, band (d) is replaced after step 1 by three new bands at 1680 (e), 1630 (f) and $1540 \mathrm{~cm}^{-1}(\mathrm{~g})$ respectively attributed to ammonium carboxylate 5 salt of Krytox ${ }^{\mathrm{TM}}$, asymmetric and symmetric $\mathrm{COO}^{-}$stretching. These bands indicate that the Krytox ${ }^{\mathrm{TM}}$ carboxylate moiety is chemisorbed onto the metal oxide through bidentate chelating interaction. $^{44-46}$
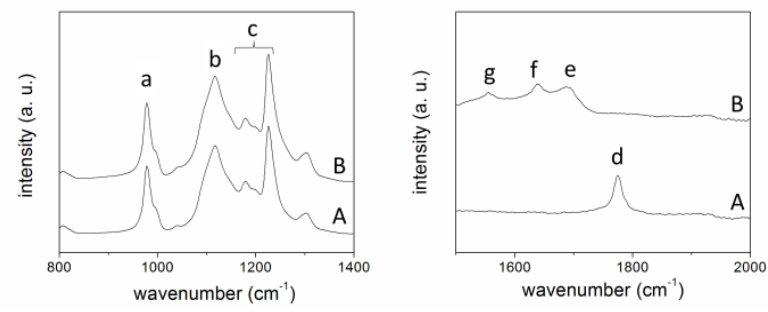

10 Fig. 5 ATR-IR spectra of Krytox ${ }^{\mathrm{TM}}$ 157-FSH (spectrum A) and of Krytox ${ }^{\mathrm{TM}}$-surfacted MNPs (spectrum B) in two wave number ranges. The letters indicate absorption peaks discussed in text.

Now comparing spectra of PFDTS before and after hydrolysis and condensation (Fig. 6: spectra C and D), the spectrum of un15 hydrolysed PFDTS silane (D) presents a group of three peaks at 2980, 2933 and $2892 \mathrm{~cm}^{-1}$ (h) that can be attributed to the C-H stretching from the $\mathrm{CH}_{2}$ and $\mathrm{O}-\mathrm{CH}_{2}-\mathrm{CH}_{3}$ groups of PFDTS. The broad peak observed at $959 \mathrm{~cm}^{-1}$ (i) is characteristic of the Si-OEt bonds. After hydrolysis-condensation, (spectrum $\mathrm{C}$ ), the 20 intensities of bands (h) and (i) decrease strongly, thus confirming almost total hydrolysis of PFDTS. Indeed, upon hydrolysis, the $\mathrm{O}-\mathrm{CH}_{2}-\mathrm{CH}_{3}$ groups initially present on the PFDTS molecules are transformed into $\mathrm{CH}_{3}-\mathrm{CH}_{2} \mathrm{OH}$ and no longer contribute to the signal of band (h). A new broad band appears at $3340 \mathrm{~cm}^{-1}$ (j) 25 corresponding to the $\mathrm{O}-\mathrm{H}$ stretching mode of $\mathrm{H}$-bonded silanol groups, which shows that condensation is not complete in the bulk system. Spectrum (C) presents three peaks at 1020, 1070 and $1112 \mathrm{~cm}^{-1}(\mathrm{k})$ that can be attributed to the formation of siloxane bonds Si-O-Si. ${ }^{47}$ The other bands observed at 1143, 1200 and $301230 \mathrm{~cm}^{-1}$ (l) are characteristic of the $-\mathrm{CF}_{2}$ stretching modes. ${ }^{48}$
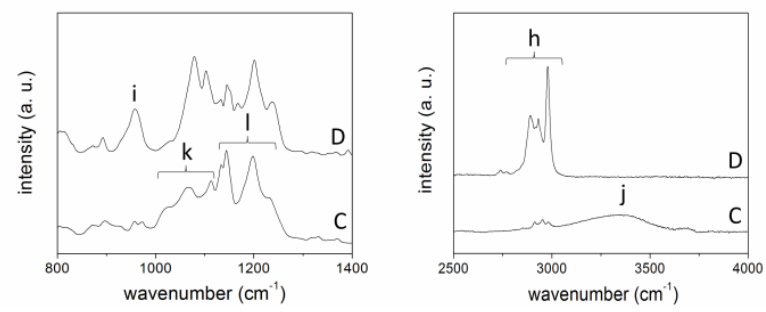

Fig. 6 ATR-IR spectra of hydrolysed and condensed PFDTS (spectrum C) and of un-hydrolysed PFDTS (spectrum D) in two wave-number ranges. The letters indicate absorption peaks discussed in text.

35 Finally, on the spectrum of 'silanized' MNPs (Fig. 7: spectrum E), the almost total disappearance of band (j) observed after grafting onto the MNPs indicates a good condensation of the silica species around the MNPs. ${ }^{49}$ Besides, the bands of $\mathrm{CH}_{2}$ groups of PFDTS are still detected (h), which indicates the 40 presence of fluorosilane at the MNPs surface. Adsorption due to fluorinated chains (1) partially overlap the band at $1070 \mathrm{~cm}^{-1}(\mathrm{k})$ associated with the formation of the Si-O-Si network. The presence of the bands at 1070 and $1112 \mathrm{~cm}^{-1}(\mathrm{k})$ indicates a high degree of condensation of silica species. ${ }^{47}$ By comparing spectra 45 (A) and (E) (pure Krytox ${ }^{\mathrm{TM}} v s$. 'silanized' MNPs), a strong decrease of the intense band (c) at $1230 \mathrm{~cm}^{-1}$ is observed compared to the spectrum of pure Krytox ${ }^{\mathrm{TM}}$. This is due to the global decrease of the number of $\mathrm{CF}_{\mathrm{x}}$ groups as the perfluoropoly(ether) Krytox ${ }^{\mathrm{TM}}$ is replaced by the much shorter ${ }_{50}$ PFDTS molecule. However, the lower but still present band (a) observed in spectrum (E) indicates that $\mathrm{Krytox}^{\mathrm{TM}}$ is still not completely removed even after several washing steps.
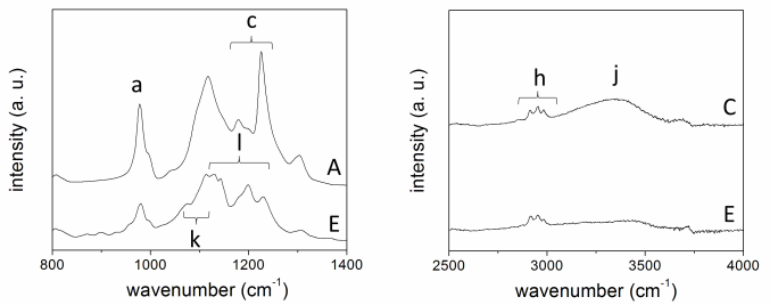

Fig. 7 ATR-IR spectra of Krytox ${ }^{\mathrm{TM}}$ 157-FSH (spectrum A), PFDTS 55 powder (spectrum C), and PFDTS-coated MNPs (spectrum E). The letters indicate absorption peaks discussed in text.

\section{XPS spectroscopy}

In order to prove the successful grafting of PFTDS onto MNPs, samples were also analysed by XPS. Fig. 8 and Fig. 9 present the ${ }_{60}$ evolution of XPS spectra of $\mathrm{C}_{1 \mathrm{~s}}$ and $\mathrm{O}_{1 \mathrm{~s}}$ sub-regions of respectively the Krytox ${ }^{\mathrm{TM}}$-surfacted and the PFDTS-silanized ferrofluids. The corresponding surface elemental concentrations are presented on Table 2.

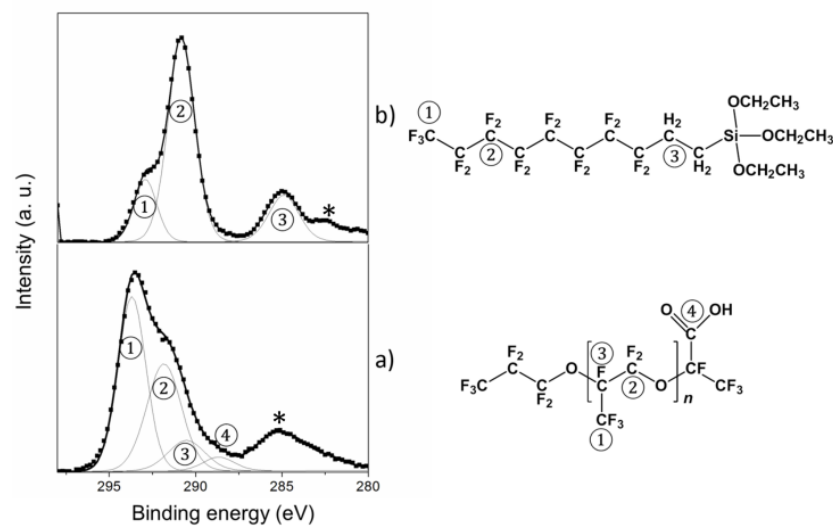

${ }_{65}$ Fig. 8 De-convoluted XPS spectra in the $\mathrm{C}_{1 \mathrm{~s}}$ region for a) the Krytox ${ }^{\mathrm{TM}}$ surfacted ferrofluid, and b) the PFDTS-silanized ferrofluid. Peaks noted with $\left(^{*}\right)$ are artefacts ascribed to satellites of the non monochromatic source of X-rays. The formulas represent molecules before reaction.

In the $\mathrm{C}_{1 \mathrm{~s}}$ region (Fig. 8a), the de-convoluted XPS spectrum of

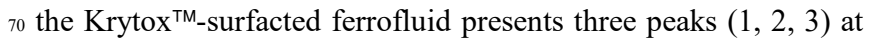
294, 292 and $291 \mathrm{eV}$ attributed respectively to carbon in $\mathrm{CF}_{3}, \mathrm{CF}_{2}$ and CF groups of Krytox ${ }^{\mathrm{TM}}$. The peak (4) at $288 \mathrm{eV}$ is assigned to carbon of carboxylate groups. After grafting of PFDTS onto the MNPs (Fig. 8b), a new peak at $285 \mathrm{eV}$ reveals the presence of 75 carbon in alkyl chains of PFDTS, ${ }^{\mathbf{5 0}}$ such $\mathrm{CH}_{2}$ groups being absent in the Krytox ${ }^{\mathrm{TM}}$ perfluoropoly(ether). The strong decrease of the intensity of the peak at $294 \mathrm{eV}$ indicates that Krytox ${ }^{\mathrm{TM}}$ is almost entirely eliminated. 
Table 2 Surface elemental concentration obtained by XPS

\begin{tabular}{cccccc} 
& \multicolumn{6}{c}{ Surface elemental concentration (\%) } \\
& $\mathrm{Si}_{2 \mathrm{p}}$ & $\mathrm{O}_{1 \mathrm{~s}}$ & $\mathrm{C}_{1 \mathrm{~s}}$ & $\mathrm{~F}_{1 \mathrm{~s}}$ & $\mathrm{Fe}_{3 \mathrm{p}}$ \\
$\gamma-\mathrm{Fe}_{2} \mathrm{O}_{3}$ control $^{a}$ & 2.06 & 51 & 27.19 & 4.34 & 15.4 \\
Surfacted-ferrofluid & 0.31 & 11.16 & 27.57 & 59.19 & 1.77 \\
Silanized-ferrofluid $^{3.2}$ & 6.22 & 29.01 & 61.24 & 0.33 \\
PFDTS powder $^{b}$ & 4.19 & 6.35 & 30.24 & 59.22 & 0
\end{tabular}

${ }^{a}$ possibly contaminated by PFDTS; ${ }^{b}$ after hydrolysis-condensation.

In the $\mathrm{O}_{1 \mathrm{~s}}$ region (Fig. 9), the XPS spectrum of Krytox ${ }^{\mathrm{TM}_{-}}$ surfacted ferrofluid exhibits two peaks at $530.2 \mathrm{eV}$ and $535.1 \mathrm{eV}$ 5 attributed respectively to oxygen in $\gamma-\mathrm{Fe}_{2} \mathrm{O}_{3}$ environment and in perfluoropropylene oxide groups of Krytox ${ }^{\mathrm{TM}}$. The deconvoluted XPS-spectrum of the $\mathrm{O}_{1 \mathrm{~s}}$ region of 'silanized' ferrofluid shows that after grafting, the $\mathrm{O}_{1 \mathrm{~s}}$ peak corresponding to oxygen from maghemite practically disappears in favour of a new peak at 532 $10 \mathrm{eV}$. This peak at $+2 \mathrm{eV}$ is generally attributed to the formation of Si-O bonds and the peak at $531.2 \mathrm{eV}$, obtained after deconvolution, is assigned to the formation of Fe-O-Si bonds. ${ }^{\mathbf{5 1}}$ The strong intensity decrease of the peak at $535 \mathrm{eV}$, assigned to the oxygen in the perfluoropoly(ether) chains, indicates that $15 \mathrm{Krytox}^{\mathrm{TM}}$ is almost entirely eliminated during the washing steps. The disappearance of the peak at $530 \mathrm{eV}$ after silanization is correlated to a silica coating around the MNPs. Moreover, the concentration of iron atoms on the surface of MNPs (Table 2) decreases strongly from 1.7 to 0.4 after grafting whereas the 20 concentration of $\mathrm{Si}$ reaches $3 \%$. The elemental concentrations measured for the silanized-ferrofluid are extremely closed to those calculated for the pure PFDTS powder. These observations are consistent with the formation of a dense silica shell, which attenuates the photoelectrons and almost totally hides the iron 25 atoms at the surface of the MNPs.

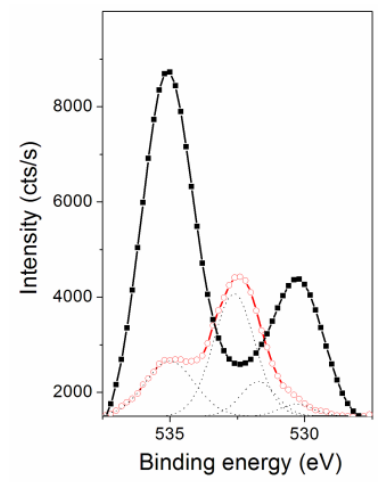

Fig. 9 XPS spectra in the $\mathrm{O}_{1 \mathrm{~s}}$ region for Krytox ${ }^{\mathrm{TM}}{ }_{\text {-surfacted ferrofluid }}$ (black line), PFDTS-silanized ferrofluid (red line) and deconvolution of XPS spectrum of PFDTS-silanized ferrofluid (dotted line).

30 The XPS and FT-IR spectroscopy results are thus in total agreement to evidence the success of the chemical grafting reaction of the fluoroalkylsilane onto the iron oxide surface.

\section{Magnetic properties of the suspensions in fluorinated oils}

The magnetisation curves of PFDTS-silanized MNPs (sample 1, 35 LP) were obtained from superconducting quantum interference device (SQUID) magnetometry measurements at 300K (Fig. 10). After subtracting the diamagnetic contribution of the solvent, the magnetisation curve $M(H)$ follows a Langevin law describing the progressive orientation of the magnetic moments along the
40 magnetic field followed by their saturation at a plateau value $M_{\mathrm{s}}$. This saturation magnetisation reads $M_{\mathrm{s}}=\phi \cdot m_{\text {spe }}$, where $m_{\text {spe }}$ is the specific magnetisation approximately equal to $3 \times 10^{5} \mathrm{~A} \cdot \mathrm{m}^{-1}$ for colloidal maghemite nanoparticles and $\phi$ is the MNPs volume fraction (the magnetisation being defined as the volume density 45 of magnetic moments). ${ }^{35}$ This superparamagnetic behaviour in the liquid state direct evidences that the silanization reaction at the surface of the MNPs enabled their stabilisation against aggregation up to a magnetic field induction as high as $7 \mathrm{~T}$.
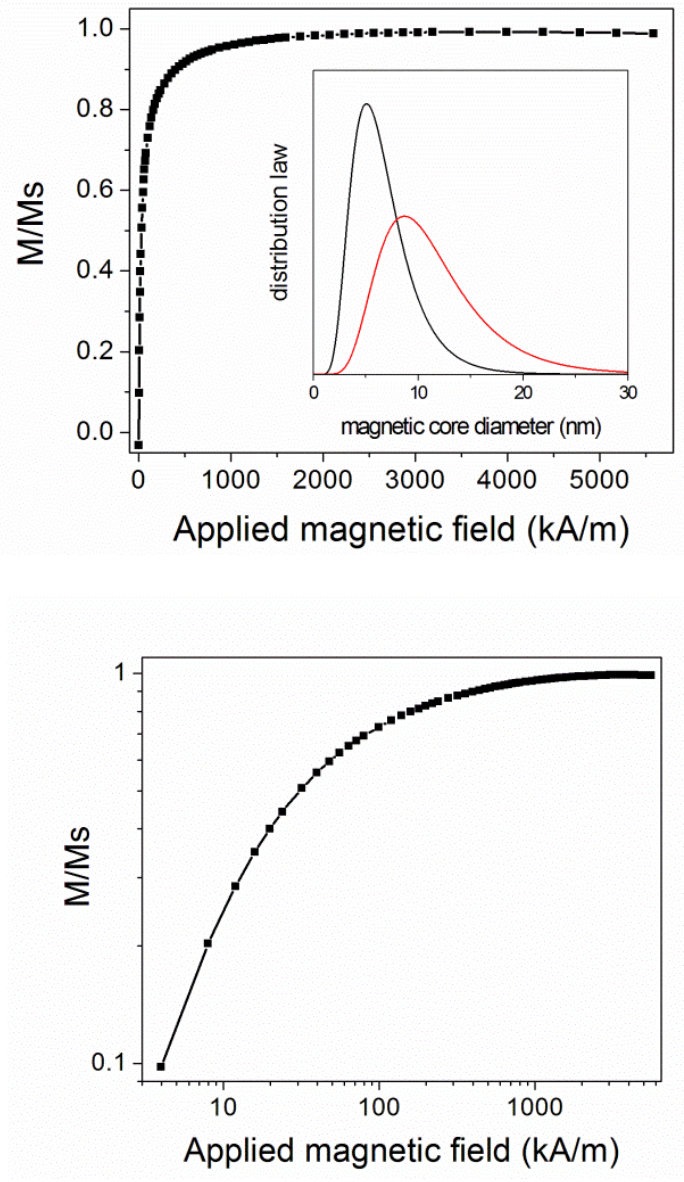

50 b)

Fig. 10 a) Magnetisation curve of the PFDTS-silanized ferrofluid sample 1 (LP) in FC-75 ( $\phi=1 \% \mathrm{v} / \mathrm{v})$ by SQUID normalised by the saturation value at high field; inset: Log-normal distribution of magnetic inorganic core diameters: by number (black line) and by volume (red line); b) 55 Magnetisation curve on a log-log plot to highlight the fit quality at low field. The value of saturation magnetisation (plateau value) is $M_{\mathrm{s}}=\phi \cdot m_{\mathrm{spe}}$ where the value of specific magnetisation is $m_{\text {spe }}=3 \times 10^{5} \mathrm{~A} \cdot \mathrm{m}^{-1}(300$ $\mathrm{emu} \cdot \mathrm{cm}^{-3}$ in CGS units) and $\phi=1 \%$ is the volume fraction of iron oxide in the suspension.

60 The magnetic susceptibility defined as the slope of the magnetisation curve at low field was $\chi=0.05$ for this sample diluted at $\phi=1 \%$. Therefore, the susceptibility of samples at any volume fraction can be calculated by $\chi=5 \cdot \phi$. The exact shape of the measured magnetisation curve $M(H)$ could be fitted by the ${ }_{65}$ convolution of the Langevin law of superparamagnetism with a size distribution of the MNPs for which we assume a log-normal law $^{\mathbf{3 5}, \mathbf{5 2}}$ as written on eqn (1). By this method, we obtained a median diameter $d_{0}{ }^{\text {SQUID }}=6.1 \mathrm{~nm}$ and a logarithmic size width 
$\sigma^{\text {SQUID }}=0.42$. The inset of Fig. 10 a) shows the particle size distribution by number and by volume. The number-averaged diameter $d_{\mathrm{n}}^{\text {SQUID }}=6.7 \mathrm{~nm}$ is determined by eqn (2), whereas the volume-averaged diameter $d_{\mathrm{w}}^{\text {SQUID }}=11.4 \mathrm{~nm}$ is calculated by eqn 5 (3). These two values are both smaller than those obtained by TEM images analysis $\left(d_{\mathrm{n}}^{\text {TEM }}=10.2 \mathrm{~nm}\right.$ and $\left.d_{\mathrm{w}}^{\text {TEM }}=19.1 \mathrm{~nm}\right)$. This difference is ascribed to the reported presence of a nonmagnetic dead layer around ferrite $\mathrm{MNPs}^{53-55}$ and to the silicon-rich shell that is visible on the TEM images but does not 10 contribute to the magnetic properties.

\section{Preparation of monodisperse magnetic emulsions as medium} for acoustic measurements

To evidence great potential applications of silanized fluorinated ferrofluids to control the propagation of ultrasounds, we prepared 15 narrowly size-dispersed emulsions in a water-based Carbopol ${ }^{\mathrm{TM}}$ yield-stress matrix, whose interest is to oppose to the settlement of droplets by gravity (fluorinated oils being much denser than aqueous media). We show here results obtained with an emulsion prepared with the LP 'silanized' ferrofluid (sample 1) composed 20 of MNPs with a hydrodynamic diameter of $51 \mathrm{~nm}(P D I=0.05)$. TEM micrographs of 'silanized' sample 1 showed magnetic core sizes of $9.3 \pm 3.4 \mathrm{~nm}$. A volume fraction of maghemite $\phi=6 \%$ in the ferrofluid was determined using UV-spectroscopy and mass density measurement. To check the ability of the ferrofluid 25 droplets to be deformed by a magnetic field, droplets of diameter around $200 \mu \mathrm{m}$ were suspended in the $\mathrm{Carbopol}^{\mathrm{TM}}$ gel at $0.05 \%$ w/w.

\section{Response of a single droplet to an applied magnetic field}

The droplet elongation under a magnetic field is characterised by 30 its aspect ratio $a / b$, where $a$ and $b$ are respectively the long and short axes of the ellipsoidal shape, measured as a function of the applied induction $B$. From Fig. 11a, it appears that the aspect ratio can reach up to 2.5 for an external field induction $B$ of $45 \mathrm{mT}$.

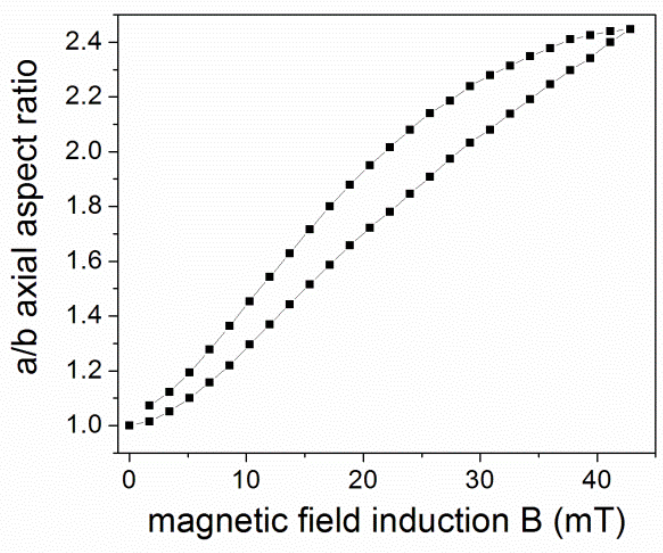

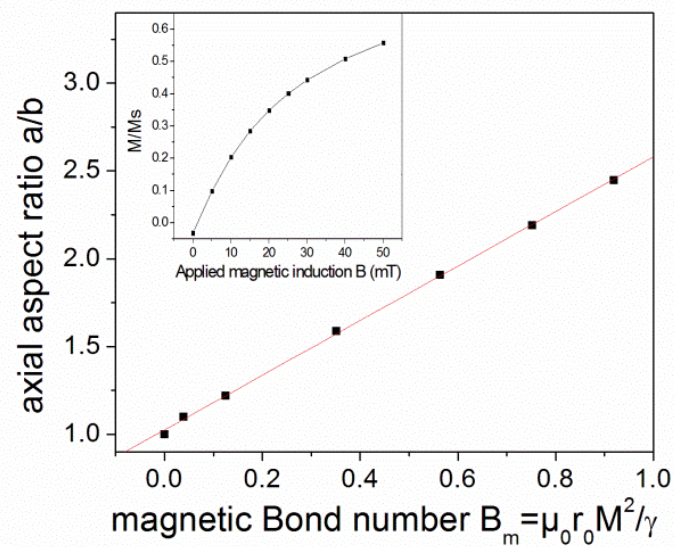

35 b)

Fig. 11 a) Evolution of the axial aspect ratio $a / b$ of a droplet of the PFDTS-silanized ferrofluid suspended in a water-based gel, of initial radius $r_{0}=104 \mu \mathrm{m}$, under increasing values of magnetic induction $B$. The apparent hysteresis when the field is decreased 40 back to zero is ascribed to slow relaxation of the gel medium (typically $30 \mathrm{~s}$ ) when the magnetic pressure is released; b) Variation of the axial aspect ratio $a / b$ of the fluorinated ferrofluid droplet represented as a function of the magnetic Bond number $B_{\mathrm{m}}$, defined as 4 times the ratio of the magnetic pressure acting on 45 the interface at the two poles by the Laplace pressure of the initially spherical droplet. The linear fit (slope $\pi / 2$, intercept 1 ) is a theoretical prediction at low deformation. ${ }^{\mathbf{5 6}}$ Inset: enlargement at low field of the reduced magnetization relatively to the saturation $M / M_{\mathrm{s}} v s$. induction $B$. This reduced plot of the SQUID 50 data measured at a $\phi=1 \%$ enables calculating the magnetization at any volume fraction $\phi$ of the same magnetic nanoparticles, in particular the emulsion droplet in the gel using the calculated saturation magnetization $M_{\mathrm{s}}=\phi \cdot m_{\mathrm{spe}}$ with $m_{\mathrm{spe}}=3 \times 10^{5} \mathrm{~A} \cdot \mathrm{m}^{-1}$, e.g. $M_{\mathrm{s}}=1.8 \times 10^{4} \mathrm{~A} \cdot \mathrm{m}^{-1}$ for $\phi=6 \%$.

55 The deformation of an isolated ferrofluid droplet by a magnetic field was studied several decades ago both theoretically ${ }^{\mathbf{5 6}}$ and experimentally. ${ }^{57}$ The model is very robust and has been verified by experimental studies on aqueous biphasic ferrofluids ${ }^{\mathbf{5 8}, 59}$ and on non aqueous, e.g. silicone, ${ }^{\mathbf{6 0}}$ ferrofluid droplets. Figure $11 \mathrm{~b}$ 60 shows another representation of the axial aspect ratio $a / b$ of the ferrofluid droplet as a function of the magnetic Bond number $B_{\mathrm{m}}$. This adimensional number compares the magnetic pressure $P_{\mathrm{m}}$ acting on the interface between the ferrofluid droplet and the non magnetic gel to the Laplace pressure $\Delta P_{\mathrm{L}}$ arising from the ${ }_{65}$ curvature of the initial droplet:

$$
B_{\mathrm{m}}=4 \frac{P_{\mathrm{m}}}{\Delta P_{\mathrm{L}}}=\frac{\mu_{0} r_{0} M^{2}}{\gamma}
$$

where $\mu_{0}=4 \pi \times 10^{-7} \mathrm{~kg} \cdot \mathrm{m} \cdot \mathrm{A}^{-2} \cdot \mathrm{s}^{-2}$ is the magnetic permeability of vacuum, $\gamma$ is the interfacial tension between the ferrofluid and the surrounding fluid (here the aqueous 70 yield-stress gel), and the values of magnetisation $M$ were obtained from the SQUID measurement curve $M / M_{\mathrm{s}} v s . B$ at low field (inset of Fig. 11b) with $M_{\mathrm{s}}=\phi \cdot m_{\text {spe }}$ where $\phi=6 \%$ and $m_{\text {spe }}=3 \times 10^{5} \mathrm{~A} \cdot \mathrm{m}^{-1}$.

The droplet radius $r_{0}=(3 \mathrm{~V} / 4 \pi)^{1 / 3}$ is measured at $104 \mu \mathrm{m}$ 75 from the volume $V$ that remained constant within $1 \%$ when plotting $V=4 \pi \mathrm{a} \cdot \mathrm{b}^{2} / 3$ whatever the applied field strength (data not shown). The values of $B_{\mathrm{m}}$ were calculated using $M$ values corresponding to each magnetic field applied to elongate the 
droplet of PFDTS-silanized ferrofluid. We observed that the axial aspect ratio $a / b$ varies perfectly linearly with $B_{\mathrm{m}}$. Such linearity is generally observed at low $B_{\mathrm{m}}$ values, typically for aspect ratios below $3 .^{57}$ Introducing a theory of the 5 deformation of magnetic droplets at any $B_{\mathrm{m}}$ values, Tsebers ${ }^{56}$ showed that at low deformation, when the eccentricity of the ellipsoidal shape, defined by $e=\sqrt{1-b^{2} / a^{2}}$, tends towards zero, then the magnetic Bond number varies as:

$$
B_{\mathrm{m}} \approx e^{2} / \pi
$$

10 When $e \rightarrow 0$, one approximates $a / b=\left(1-\mathrm{e}^{2}\right)^{-0.5} \approx 1+0.5 \mathrm{e}^{2}$, thus we deduce the equation giving the aspect ratio variation in the linear regime:

$$
a / b \approx 1+\frac{\pi}{2} B_{\mathrm{m}}
$$

By fitting the experimental plot of $a / b v s . B_{\mathrm{m}}$ with a line of 15 slope $\pi / 2$ and intercept 1 , we get the value $\gamma=11.8 \mathrm{mN} \cdot \mathrm{m}^{-1}$ for the interfacial tension, in excellent agreement with the interfacial tension $\gamma=11 \mathrm{mN} \cdot \mathrm{m}^{-1}$ measured by the pendant drop method. The Laplace pressure difference $\Delta P_{\mathrm{L}}=2 \gamma / r_{0}$ between the interior of the initially spherical droplet and the 20 gel is estimated at $\Delta P_{\mathrm{L}}=230 \mathrm{~Pa}$ with the interfacial tension deduced previously. Therefore, even if the applied field is at its lowest value corresponding to $B_{\mathrm{m}}=0.04$, the pressure acting on the magnetic poles of the droplet is estimated at $P_{m}$ $\approx 0.01 \times 230=2.3 \mathrm{~Pa}$. This value is slightly above the yield ${ }_{25}$ stress of the gel (measured value of $1.5 \mathrm{~Pa}$ ). We can thus conclude that the applied magnetic pressure overcomes the yield stress of the water-based gel, which allows fluid motion around the droplet accompanying its deformation. In the opposite situation when the droplet is elongated and the 30 magnetic field is switched off, the slow shape relaxation kinetics can be ascribed to a pressure gap between the poles and the equator of the ellipsoid lower than $P_{\mathrm{m}}$ acting when the field was on, but still sufficient to cause the flow of the gel (i.e. above the yield stress).

\section{Monodisperse magnetic emulsions in water-based gel}

The silanized fluorinated ferrofluid was injected through a silica capillary (with internal diameter $I D$ of 40 or $75 \mu \mathrm{m}$ ) into the gel $\left(\right.$ Carbopol $\left.^{\mathrm{TM}} 0.05 \% \mathrm{w} / \mathrm{w}\right)$ under a controlled pressure. ${ }^{14,} 38$ The deposition was obtained by motorised translation of the plate at a 40 speed of $1 \mathrm{~mm} \cdot \mathrm{s}^{-1}$ (Fig. 12) for the smaller capillary or $10 \mathrm{~mm} \cdot \mathrm{s}^{-1}$ for the larger one. The yield stress of the Carbopol ${ }^{\mathrm{TM}}$ gel was measured at $1.5 \mathrm{~Pa}$ for a concentration of $0.05 \% \mathrm{w} / \mathrm{w}$ by standard rheological measurement (cone-plan AR2000 rheometer). We calculated that fluorinated ferrofluid droplets (mass density $d=$ 45 $1.9 \mathrm{~g} \cdot \mathrm{cm}^{-3}$ ) can stay suspended as long as their diameter remains lower than $250 \mu \mathrm{m}$ (balancing the hydrostatic pressure $P_{\mathrm{h}}=$ $2 / 3 r_{0} \cdot g \cdot \rho$ with the yield stress). The sizes of the emulsion droplets were measured on optical images. The mean diameter and coefficient of variation $C V$ (ratio of the standard width to the ${ }_{50}$ mean value, assuming Gaussian distribution) were deduced from these measurements on a large statistics. The conclusion is that nearly monodisperse magnetic emulsions can be produced by this method, an example being presented on Fig. 12.
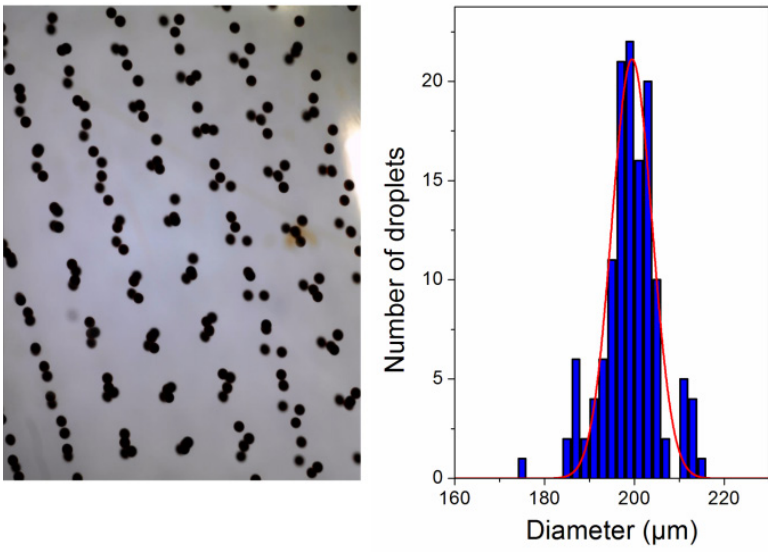

55 Fig. 12 Optical micrograph and corresponding size histogram deduced from image analysis of a magnetic fluorinated emulsion. Volume fraction $\approx 0.5 \%$ of the matrix $\left(0.05 \% \mathrm{w} / \mathrm{w}\right.$ Carbopol $\left.^{\mathrm{TM}}\right)$. Droplets containing $\phi=$ $6 \% \mathrm{v} / \mathrm{v}$ of MNPs ('silanized' sample 1) were deposited through a capillary of $I D=40 \mu \mathrm{m}$ under a $1.7 \mathrm{bar}$ air pressure, translated at a speed $60 \mathrm{of} 1 \mathrm{~mm} \cdot \mathrm{s}^{-1}$. The distribution yielded an average diameter of $199 \mu \mathrm{m}$ with $C V=3.2 \%$ (measured on 133 droplets).

\section{Acoustic properties}

These emulsions were analysed by multiple-echo ultrasound spectroscopy acquired with a piezoelectric transducer of $5 \mathrm{MHz}$ ${ }_{65}$ central frequency stuck directly on the measurement cell. Fig. 13 shows the evolution of the attenuation coefficient vs. frequency curves for magnetic inductions of 0,20 , and $40 \mathrm{mT}$. Under a zero magnetic field, the attenuation spectrum exhibits several peaks at specific frequencies, in accordance with previous results obtained 70 for pure (non magnetic) fluorocarbon emulsions of $C V \approx 5 \% .^{38}$ These attenuation peaks result from Mie resonances created by the sound-speed contrast existing between the host matrix and the fluorinated ferrofluid droplets.

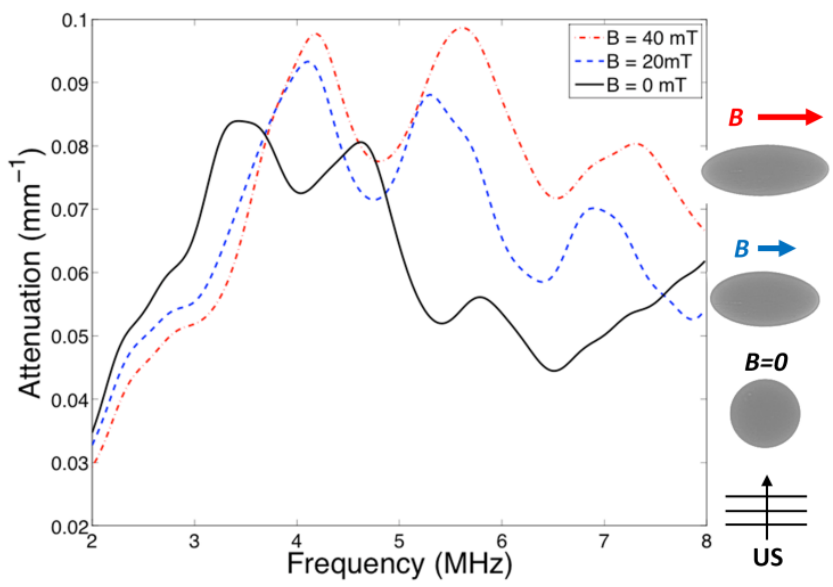

75 Fig. 13 Acoustic spectrum (attenuation coefficient vs. wave frequency) for increasing magnetic field induction values $B(\mathrm{mT})$ applied perpendicularly to the propagation direction of the ultrasound wave.

When a magnetic field was applied perpendicularly to the direction of acoustic wave propagation, these attenuation peaks 80 were shifted towards higher frequencies. This evolution is explained by the change of the shape of the ferrofluid droplets from spheres to ellipsoids, in that case elongated perpendicularly to the US wave propagation vector. Qualitatively, the dimension 
of the droplets probed by the ultrasounds becomes smaller, thus the resonance frequencies become higher. A quantitative description of this effect with a complete fitting of the spectra under varying magnetic field strengths and directions is the 5 subject of another article. ${ }^{15}$

\section{Conclusions}

In summary, we described a chemical route based on aqueous coprecipitation synthesis, perfluoropoly(ether) surfactant coating, ligand exchange and sol-gel reaction to achieve a stable 10 fluorinated ferrofluid. After silanization, the MNPs coated by a thin fluorinated silica shell can be dispersed individually in any fluorinated oil. The obtained dispersions of silanized-MNPs exhibiting a high colloidal stability and low viscosity were used to prepare monodisperse emulsions in a water-based Bingham gel 15 with a microfluidic injection device. The lower viscosity compared to a mere 'surfacted' ferrofluid is ascribed to the replacement of the macromolecular surfactant with a thin layer of much shorter perfluorosilane (since in the denomination Krytox ${ }^{\mathrm{TM}}$ 157-FSH, the H letter means "high viscosity"). Concerning the 20 decrease of interfacial tension $\gamma$ with the aqueous phase, this might be a consequence of the ligand-exchange that causes desorption of the surfactant from the iron oxide surface and adsorption of a small fraction of Krytox ${ }^{\mathrm{TM}}$ still remaining after the washing steps (as seen by FT-IR and XPS spectroscopy) at the

25 fluorinated oil/water interface. Such a reducing effect of $\gamma$ was indeed also noticed with another fluorinated surfactant, Dupont Zonyl $^{\text {TM }}$ FSO (data not shown).

The droplet shape elongates along the direction of the applied magnetic field. These reversible deformations can be perfectly 30 controlled by modulating the magnetic field induction. Acoustic measurements have shown that these emulsions behave as a "resonant material" exhibiting attenuation peaks at specific frequencies in the Mie scattering regime. By applying a magnetic field, we can tune the resonant acoustic response of such a 35 scattering medium in a reversible manner. By varying the magnetic field from to 0 to $40 \mathrm{mT}$, we can precisely control the attenuation frequency and the attenuation coefficient of the material. The droplets described here have a radius around 100 $\mu \mathrm{m}$, while typical diameters of "microbubbles" used as contrast 40 agents in ultra-sonography are rather in the $1-10 \mu \mathrm{m}$ range, i.e. not larger than red blood cells, to avoid clogging of blood vessels or kidney. Nevertheless, magnetic emulsions allow convenient size selection using a method based on their sensitivity to an external magnetic field, by forming dipolar chains preferably 45 made of droplets of analogous diameter, and that can be separated by a field strength gradient. ${ }^{\mathbf{6 1}, \mathbf{6 2}}$ Starting from a polydisperse magnetic emulsion, this size-grading method can lead to monodisperse emulsions with target sizes ranging from the submicrometric up to the millimetric range, thus opening the way to 50 acoustic applications from the audible $(20 \mathrm{~Hz}-20 \mathrm{kHz})$ to the US regime $(100 \mathrm{kHz}-10 \mathrm{MHz})$. The locally resonant emulsions described in this article appear to be the constitutive brick elements for the preparation of tuneable acoustic materials. ${ }^{63}$

\section{Notes and references}

${ }_{55}{ }^{1}$ Université de Bordeaux, CRPP / CNRS UPR 8641 Centre de Recherche Paul Pascal, 115 avenue Schweitzer, 33600 Pessac, France.
${ }^{2}$ Université de Bordeaux, LCPO / CNRS UMR 5629 Laboratoire de Chimie des Polymères Organiques, 16 avenue Pey Berland, 33607 Pessac, France.

$60{ }^{3}$ Université de Bordeaux, I2M-APY / CNRS UMR 5295 Institut de Mécanique et d'Ingénierie, 351 cours de la libération, 33405 Talence, France.

${ }^{4}$ Université de Bordeaux, LOF / Solvay / CNRS UMR 5258 Laboratory of Future, 178 avenue Schweitzer, 33600 Pessac, France.

$65 \dagger$ Electronic Supplementary Information (ESI) available: complete XPS spectra and movies of the deformation of a single fluorinated ferrofluid droplet embedded in a water-based gel matrix submitted to the magnetic field 1) created by a permanent magnet; 2 ) by a solenoid producing an increasing magnetic induction between $B=0$ and $B=46 \mathrm{mT}$. See 70 DOI: $10.1039 / \mathrm{C} 3 \mathrm{~TB} 21585 \mathrm{G}$

$\$$ These authors contributed equally.* Please send any correspondance to: olivier.sandre@enscbp.fr, mondain@crpp-bordeaux.cnrs.fr.

\section{Aknowledgments}

This work was supported by the US Air Force European Office of 75 Aerospace Research and Development (EOARD Grants FA8655-11-M4006 and FA8655-12-1-2067), by the Agence Nationale de la Recherche (ANR Grant 2011-BS0902101), by the Conseil Régional d'Aquitaine and the Polytechnic Institute of Bordeaux. We thank Rodolphe Clérac and Mathieu Rouzieres at CRPP (UPR8641 CNRS/Université de Bordeaux) 80 for the SQUID curves and Christine Labrugère at ICMCB (UPR9048 CNRS/Université de Bordeaux) for the XPS spectra.

\section{Abbreviations}

ATR, attenuated total reflection; CV, coefficient of variation; DLS, dynamic light scattering; FT-IR, Fourier transform infrared spectroscopy; ${ }_{85} \mathrm{ID}$, internal diameter; LP, large particles; MNP, magnetic nanoparticle; OD, outer diameter; PDI, polydispersity index; PFDTS, perfluorodecyltriethoxysilane; PFOA, perfluorooctanoic acid; PFOB, perfluorooctyl bromide; SANS, small angle neutron scattering; SLD, scattering length density; SP, small particles; SQUID, superconducting 90 quantum interference device; TEM, transmission electron microscopy; TMAOH, tetramethylammonium hydroxide; UCA, ultrasound contrast agents; US, ultrasound; XPS, X-ray photoelectron spectroscopy.

1. R. Berger, G. Resnati, P. Metrangolo, E. Weber and J. Hulliger, 95 Chemical Society Reviews, 2011, 40, 3496-3508.

2. R. C. Buck, J. Franklin, U. Berger, J. M. Conder, I. T. Cousins, P. de Voogt, A. A. Jensen, K. Kannan, S. A. Mabury and S. P. J. van Leeuwen, Integrated Environmental Assessment and Management, 2011, 7, 513-541.

100 3. N. Kudo and Y. Kawashima, The Journal of Toxicological Sciences, 2003, 28, 49-57.

4. A. B. Lindstrom, M. J. Strynar and E. L. Libelo, Environmental Science \& Technology, 2011, 45, 7954-7961.

5. E. G. Schutt, D. H. Klein, R. M. Mattrey and J. G. Riess, Angewandte 105 Chemie International Edition, 2003, 42, 3218-3235.

6. E. Stride and M. Edirisinghe, Soft Matter, 2008, 4, 2350-2359.

7. O. Diou, N. Tsapis, C. 1. Giraudeau, J. Valette, C. Gueutin, F. Bourasset, S. Zanna, C. Vauthier and E. Fattal, Biomaterials, 2012, 33, 5593-5602.

110 8. S. Rossi, G. Waton and M. P. Krafft, Langmuir, 2009, 26, 16491655.

9. S. Rossi, C. Szijjarto, F. Gerber, G. Waton and M.-P. Krafft, Journal of Fluorine Chemistry, 2011, 132, 1102-1109.

10. D. Vlaskou, O. Mykhaylyk, F. Krötz, N. Hellwig, R. Renner, U. 115 Schillinger, B. Gleich, A. Heidsieck, G. Schmitz, K. Hensel and C. Plank, Advanced Functional Materials, 2010, 20, 3881-3894.

11. A. L. Klibanov, T. I. Shevchenko, B. I. Raju, R. Seip and C. T. Chin, Journal of Controlled Release, 2010, 148, 13-17. 
12. J. Owen, B. Zhou, P. Rademeyer, M.-X. Tang, Q. Pankhurst, R. Eckersley and E. Stride, Theranostics, 2012, 2, 1127-1139.

13. P. N. Nguyen, G. Nikolova, P. Polavarapu, G. Waton, L. T. Phuoc, G. Pourroy and M. P. Krafft, RSC Advances, 2013, 3, 7743-7746.

5 14. T. Brunet, S. Raffy, B. Mascaro, J. Leng, R. Wunenburger, O. Mondain-Monval, O. Poncelet and C. Aristégui, Applied Physics Letters, 2012, 101, 011913.

15. T. Brunet, K. Zimny, B. Mascaro, O. Sandre, O. Poncelet, C. Aristégui and O. Mondain-Monval, Physical Review Letters, 2013,

$10 \quad \mathbf{1 1 1}, 264301$.

16. H.-C. Zhou, J. R. Long and O. M. Yaghi, Chemical Reviews, 2012, 112, 673-674.

17. C. B. Kristalyn, S. Watt, S. A. Spanninga, R. A. Barnard, K. Nguyen and Z. Chen, Journal of Colloid and Interface Science, 2011, 353,

$15 \quad 322-330$.

18. K. Tsujii, T. Yamamoto, T. Onda and S. Shibuichi, Angewandte Chemie International Edition in English, 1997, 36, 1011-1012.

19. Z. Xu, Q. Liu and J. A. Finch, Applied Surface Science, 1997, 120, 269-278.

20 20. M. Yamaura, R. L. Camilo, L. C. Sampaio, M. A. Macêdo, M. Nakamura and H. E. Toma, Journal of Magnetism and Magnetic Materials, 2004, 279, 210-217.

21. A. del Campo, T. Sen, J.-P. Lellouche and I. J. Bruce, Journal of Magnetism and Magnetic Materials, 2005, 293, 33-40.

25 22. S. Mornet, J. Portier and E. Duguet, Journal of Magnetism and Magnetic Materials, 2005, 293, 127-134.

23. I. J. Bruce and T. Sen, Langmuir, 2005, 21, 7029-7035.

24. W. Wu, Q. He and C. Jiang, Nanoscale Research Letters, 2008, 3, 397-415.

30 25. F. Galeotti, F. Bertini, G. Scavia and A. Bolognesi, Journal of Colloid and Interface Science, 2011, 360, 540-547.

26. N. Arsalani, H. Fattahi, S. Laurent, C. Burtea, L. V. Elst and R. N. Muller, Contrast Media \& Molecular Imaging, 2012, 7, 185-194.

27. T. Borase, T. Ninjbadgar, A. Kapetanakis, S. Roche, R. O'Connor, C.

35 Kerskens, A. Heise and D. F. Brougham, Angewandte Chemie International Edition, 2013, 52, 3164-3167.

28. R. De Palma, S. Peeters, M. J. Van Bael, H. Van den Rul, K. Bonroy, W. Laureyn, J. Mullens, G. Borghs and G. Maes, Chemistry of Materials, 2007, 19, 1821-1831.

40 29. J. Trekker, K. Jans, H. Damm, D. Mertens, T. Nuytten, J. Vanacken, V. Moshchalkov, J. D'Haen, T. Stakenborg, W. Van Roy, U. Himmelreich and L. Lagae, Magnetics, IEEE Transactions on, 2013, 49, 219-226.

30. N. Kohler, G. E. Fryxell and M. Zhang, Journal of the American

$45 \quad$ Chemical Society, 2004, 126, 7206-7211.

31. T. Ninjbadgar and D. F. Brougham, Advanced Functional Materials, 2011, 21, 4769-4775.

32. D. Forge, S. Laurent, Y. Gossuin, A. Roch, L. Vander Elst and R. N. Muller, Journal of Magnetism and Magnetic Materials, 2011, 323, $50 \quad 410-415$.

33. Y. Sun, X. Ding, Z. Zheng, X. Cheng, X. Hu and Y. Peng, European Polymer Journal, 2007, 43, 762-772.

34. R. Massart, Magnetics, IEEE Transactions on, 1981, 17, 1247-1248.

35. R. Massart, E. Dubois, V. Cabuil and E. Hasmonay, Journal of

55 Magnetism and Magnetic Materials, 1995, 149, 1-5.
36. K. L. O'Neal, H. Zhang, Y. Yang, L. Hong, D. Lu and S. G. Weber, Journal of Chromatography A, 2010, 1217, 2287-2295.

37. V. Leroy, A. Strybulevych, J. H. Page and M. G. Scanlon, The Journal of the Acoustical Society of America, 2008, 123, 1931-1940.

60 38. B. Mascaro, T. Brunet, O. Poncelet, C. Aristégui, S. Raffy, O. Mondain-Monval and J. Leng, The Journal of the Acoustical Society of America, 2013, 133, 1996-2003.

39. A. Galimard, M. Safi, N. Ould-Moussa, D. Montero, H. Conjeaud and J.-F. Berret, Small, 2012, 8, 2036-2044.

${ }_{65}$ 40. P. Arosio, J. Thévenot, T. Orlando, F. Orsini, M. Corti, M. Mariani, L. Bordonali, C. Innocenti, C. Sangregorio, H. Oliveira, S. Lecommandoux, A. Lascialfari and O. Sandre, Journal of Materials Chemistry B, 2013, 1, 5317-5328.

41. A. Brulet, D. Lairez, A. Lapp and J.-P. Cotton, Journal of Applied 70 Crystallography, 2007, 40, 165-177.

42. I. P. Vinogradov, A. Dinkelmann and A. Lunk, Surface and Coatings Technology, 2003, 174-175, 509-514.

43. L. Zhang, R. He and H.-C. Gu, Applied Surface Science, 2006, 253, 2611-2617.

75 44. Y. Ren, K.-i. limura and T. Kato, Langmuir, 2001, 17, 2688-2693.

45. N. Wu, L. Fu, M. Su, M. Aslam, K. C. Wong and V. P. Dravid, Nano Letters, 2004, 4, 383-386.

46. A. L. Willis, N. J. Turro and S. O'Brien, Chemistry of Materials, 2005, 17, 5970-5975.

80 47. A. Vilcnik, I. Jerman, A. Surca Vuk, M. Kozelj, B. Orel, B. Tomsic, B. Simoncic and J. Kovac, Langmuir, 2009, 25, 5869-5880.

48. T. J. Lenk, V. M. Hallmark, C. L. Hoffmann, J. F. Rabolt, D. G. Castner, C. Erdelen and H. Ringsdorf, Langmuir, 1994, 10, 46104617.

85 49. J. L. Blin and C. Carteret, The Journal of Physical Chemistry C, 2007, 111, 14380-14388.

50. A. Chandekar, S. K. Sengupta and J. E. Whitten, Applied Surface Science, 2010, 256, 2742-2749.

51. M. R. Turner, E. Duguet and C. Labrugère, Surface and Interface 90 Analysis, 1997, 25, 917-923.

52. R. W. Chantrell, J. Popplewell and S. Charles, Magnetics, IEEE Transactions on, 1978, 14, 975-977.

53. K. Haneda and A. H. Morrish, Magnetics, IEEE Transactions on, 1980, 16, 50-52.

95 54. Y.-w. Jun, J.-w. Seo and J. Cheon, Accounts of Chemical Research, 2008, 41, 179-189.

55. M. P. Morales, S. Veintemillas-Verdaguer, M. I. Montero, C. J. Serna, A. Roig, L. Casas, B. Martínez and F. Sandiumenge, Chemistry of Materials, 1999, 11, 3058-3064.

100 56. A. O. Tsebers, Magnetohydrodynamics, 1985, 21, 19-26.

57. V. I. Arkhipenko, Y. D. Barkov and V. G. Bashtovoi, Magnetohydrodynamics, 1978, 14, 373-375.

58. J. C. Bacri and D. Salin, J. Physique Lett., 1982, 43, 649-654.

59. O. Sandre, J. Browaeys, R. Perzynski, J. C. Bacri, V. Cabuil and R. 105 E. Rosensweig, Physical Review E, 1999, 59, 1736-1746.

60. S. Afkami, A. J. Tyler, Y. Renardy, M. Renardy, T. G. St. Pierre, R. C. Woodward and J. S. Riffle, Journal of Fluid Mechanics, 2010, 663, 358-384.

61. J. Bibette, Journal of Magnetism and Magnetic Materials, 1993, 122, $110 \quad 37-41$. 
62. F. Montagne, O. Mondain-Monval, C. Pichot, H. Mozzanega and A. Elaissari, Journal of Magnetism and Magnetic Materials, 2002, 250, 302-312.

63. T. Brunet, J. Leng and O. Mondain-Monval, Science, 2013, 342, 3235324. 